

\title{
Neutrino Physics from the Cosmic Microwave Background and Large Scale Structure
}

\author{
Topical Conveners: K.N. Abazajian, J.E. Carlstrom, A.T. Lee \\ K.N. Abazajian*, K. Arnold, J. Austermann, B.A. Benson, C. Bischoff, J. Bock, J.R. Bond, J. Borrill, \\ E. Calabrese, J.E. Carlstrom, C.S. Carvalho, C.L. Chang, H.C. Chiang, S. Church, A. Cooray, \\ T.M. Crawford, K.S. Dawson, S. Das, M.J. Devlin, M. Dobbs, S. Dodelson, O. Doré, J. Dunkley, J. Errard, \\ A. Fraisse, J. Gallicchio, N.W. Halverson, S. Hanany, S.R. Hildebrandt, A. Hincks, R. Hlozek, G. Holder, \\ W.L. Holzapfel, K. Honscheid, W. Hu, J. Hubmayr, K. Irwin, W.C. Jones, M. Kamionkowski, B. Keating, \\ R. Keisler, L. Knox, E. Komatsu, J. Kovac, C.-L. Kuo, C. Lawrence, A.T. Lee, E. Leitch, E. Linder, \\ P. Lubin, J. McMahon, A. Miller, L. Newburgh, M.D. Niemack, H. Nguyen, H.T. Nguyen, L. Page, \\ C. Pryke, C.L. Reichardt, J.E. Ruhl, N. Sehgal, U. Seljak, J. Sievers, E. Silverstein, A. Slosar, K.M. Smith, \\ D. Spergel, S.T. Staggs, A. Stark, R. Stompor, A.G. Vieregg, G. Wang, S. Watson, E.J. Wollack, \\ W.L.K. Wu, K.W. Yoon, and O. Zahn \\ *Corresponding author. Phone: +1 (949) 824-0368, Fax: +1 (949) 824-2174, email: kevork@uci.edu
}

\section{Executive Summary}

The cosmological background of neutrinos thermally produced in the big bang has been definitively (albeit indirectly) detected. Measurements of the cosmic microwave background (CMB) alone have led to a constraint on the effective number of neutrino species of $N_{\text {eff }}=3.36 \pm 0.34$ [1], a value $10 \sigma$ away from zero and consistent with expectations. Experiments planned and underway are prepared to study this background in detail via its influence on distance-redshift relations and the growth of structure. The program for the next decade described in this document, including upcoming spectroscopic surveys eBOSS and DESI and a new Stage-IV CMB polarization experiment CMB-S4, will achieve $\sigma\left(\sum m_{\nu}\right)=16 \mathrm{meV}$ and $\sigma\left(N_{\text {eff }}\right)=0.020$. Such a mass measurement will produce a high significance detection of non-zero $\sum m_{\nu}$, whose lower bound derived from atmospheric and solar neutrino oscillation data is about $58 \mathrm{meV}$. If neutrinos have a normal mass hierarchy, this measurement will definitively rule out the inverted neutrino mass hierarchy, shedding light on one of the most puzzling aspects of the Standard Model of particle physics - the origin of mass.

This precise a measurement of $N_{\text {eff }}$ will allow for a precision test of the standard cosmological model prediction that $N_{\text {eff }}=3.046$. The difference from three is due to the small amount of entropy from electron/positron annihilation that gets transferred to the neutrinos; $N_{\text {eff }}$ is by design equal to three in the idealized case that all of this entropy is transferred to photons. Finding $N_{\text {eff }}$ consistent with 3.046 would demonstrate that we understand very well the thermal conditions in the universe at just one second after the big bang. On the other hand, finding $N_{\text {eff }}$ significantly different from 3.046 would be a signature of new physics. Possibilities include sterile neutrinos produced via oscillations in the early universe, a large matter/anti- 
matter asymmetry in the neutrino sector, or new particles that interact even more weakly than neutrinos but that were in thermal and chemical equilibrium deep in the radiation-dominated era (e.g., [2]).

The effects of neutrino properties on cosmology are precisely predicted from theory, appear in many observables in diverse ways, and are clearly observationally distinguishable from the effects of other cosmological parameters. For example, increasing $N_{\text {eff }}$ not only suppresses small-scale fluctuation power in the CMB, but also leads to detectable changes in the temporal phase of the acoustic oscillations, e.g., [3]. Increasing $\sum m_{\nu}$ in a manner that is consistent with the measured CMB power spectrum leads to very specific redshiftand scale-dependent changes to the power spectrum of both matter and the galaxy distribution [4]. These signatures will provide important consistency tests.

Much of the sensitivity to $\sum m_{\nu}$ will come from measurements of the gravitational lensing of the CMB, measurements of the baryon acoustic oscillation (BAO) features and broadband power spectrum, and measurements of weak gravitational lensing of galaxies. Different combinations of these probes have completely independent systematic errors.

Significant progress has been made recently in these areas, with the first projected mass reconstructions from CMB lensing [5, 6, 7, 8, 9, 10], detection of the CMB lensing $B$-mode polarization [11], and percent level measurements of the distance scale from BAO measurements [12, 13, 14, 15].

\section{Introduction}

\subsection{Motivation}

One of the most remarkable aspects of physical cosmology is that the study of the largest physical structures in the universe can reveal the properties of the particles with the smallest known cross section: neutrinos. At its root, this cosmological sensitivity to neutrino properties is due to the fact that the neutrino cosmological number density is second only to CMB photons. Coupled with their non-zero mass, the large number of neutrinos leads to an energy density today at least 25 times larger than the CMB. This high density of free streaming particles rapidly inhibits the growth of structure at late times, leading to changes in large scale structure (LSS) that can be captured in large galaxy surveys or CMB lensing measurements.

The CMB and LSS are sensitive to the total energy density of cosmic neutrinos today, which is effectively a measure of the sum of the neutrino masses, since the number density is known (up to uncertainties in the CMB temperature and $N_{\text {eff }}$ ). These Cosmic Frontier experiments are then exactly complementary to terrestrial laboratory experiments on the Intensity Frontier that measure the differences of mass squared and $\mathrm{CP}$-violation in the neutrino sector.

Short baseline neutrino oscillation results hint at a richer neutrino sector with more than three active neutrinos participating in flavor oscillations, i.e. with one or more sterile flavors $[16,17,18,19]$. The reactor neutrino anomaly may also indicate the presence of light sterile neutrinos [20,21]. If they do indeed exist, these extra degrees of freedom would affect the cosmic neutrino background, and the relativistic energy density in the early universe. As we will discuss below, future CMB-S4 and LSS experiments in the Cosmic Frontier have the sensitivity to make a high-confidence detection of more than three standard neutrinos or new relativistic degrees of freedom in a new particle sector behaving like neutrinos.

A global fit to solar and atmospheric neutrino flavor oscillations implies a difference in the squares of masses $m_{2}^{2}-m_{1}^{2}=7.54_{-0.22}^{+0.26} \times 10^{-5} \mathrm{eV}^{2}$ and $m_{3}^{2}-\left(m_{1}^{2}+m_{2}^{2}\right) / 2= \pm 2.43_{-0.10}^{+0.06} \times 10^{-3} \mathrm{eV}^{2}$ [22]. For the minimal case of a normal hierarchy, where $m_{1}<m_{2} \ll m_{3}$, the smallest possible value for the sum of the masses occurs 


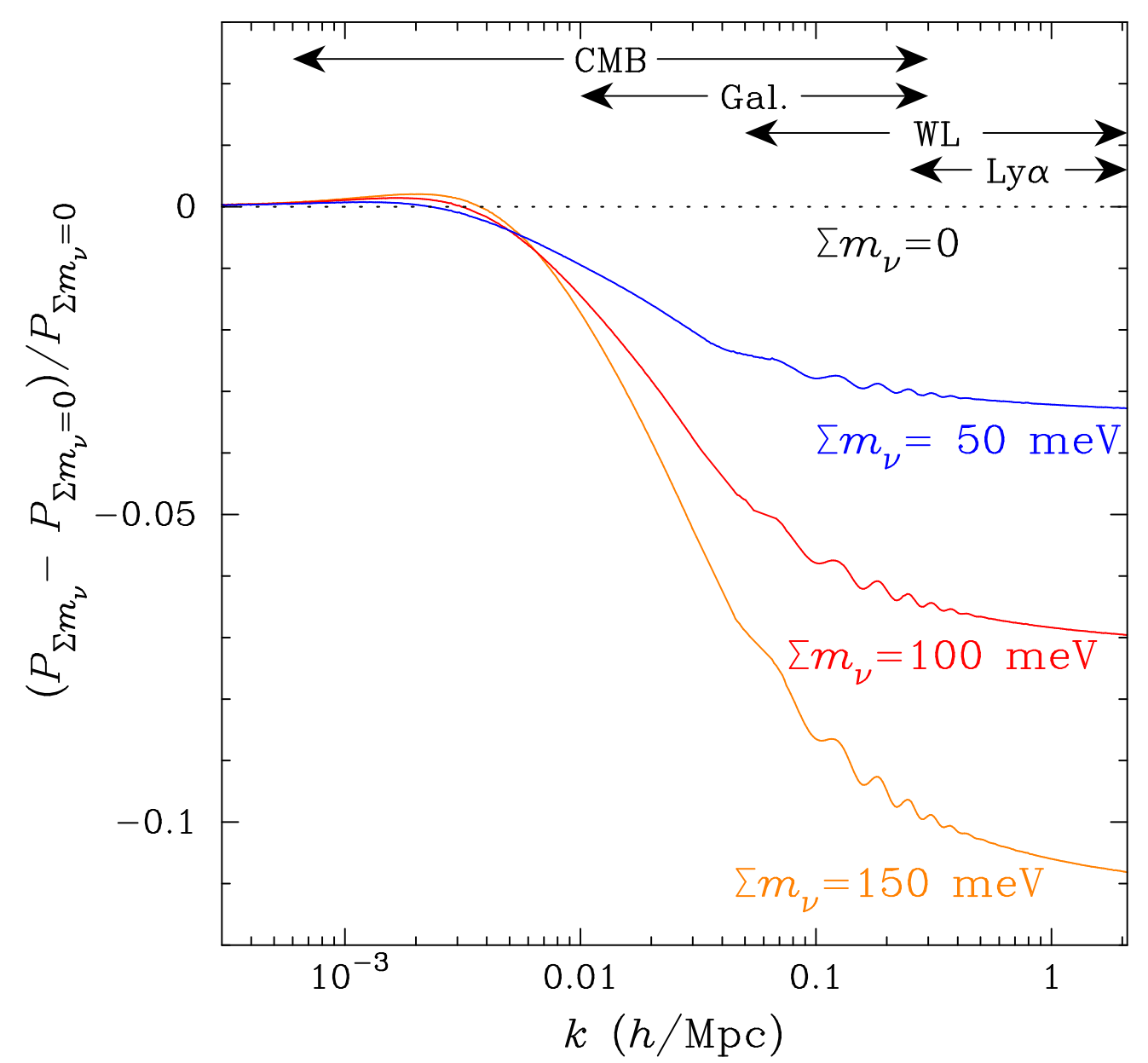

Figure 1. Fractional change in the matter density power spectrum as a function of comoving wavenumber $k$ for different values of $\sum m_{\nu}$. Neutrino mass suppresses the power spectrum due to free streaming below the matter-radiation equality scale. The shape of the suppression is highly characteristic and precision observations over a range of scales can measure the sum of neutrino masses (here assumed all to be in a single mass eigenstate). Also shown are the approximate ranges of experimental sensitivity in the power spectrum for representative probes: the cosmic microwave background (CMB), galaxy surveys (Gal.), weak

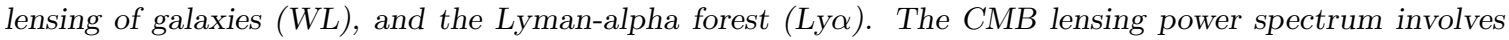
(an integral over) this same power spectrum, and so is also sensitive to neutrino mass.

when $m_{1}=0, m_{2}=8.68_{-0.13}^{+0.15} \mathrm{meV}$, and $m_{3}=49.7_{-1.0}^{+0.8} \mathrm{meV}$. So, in the normal hierarchy, the minimum sum of the masses is $\sum m_{\nu}=58.4_{-0.8}^{+1.2} \mathrm{meV}$. In the case of a so-called inverted hierarchy, where $m_{1} \simeq m_{2} \gg m_{3}$, the minimum sum of the masses must be greater than $100 \mathrm{meV}$. For the degenerate neutrino mass case where $m_{1} \simeq m_{2} \simeq m_{3}$, the sum of neutrino masses is at least approximately $150 \mathrm{meV}$. As we will discuss below, future CMB-S4 and LSS experiments in the Cosmic Frontier have projected constraints to detect the minimum mass scale of $58 \mathrm{meV}$ at $\sim 4 \sigma$ confidence, a ground-breaking result. 


\subsection{The Effective Number of Neutrino Species, $N_{\text {eff }}$}

Relic primordial neutrinos leave a distinct signature in the CMB. Assuming that all entropy produced by electron-position annihilation is transferred to photons, it can be shown, by considering the available degrees of freedom, that the temperature of the photon gas is a factor of $(11 / 4)^{1 / 3}$ higher than the temperature of the neutrino gas. The total cosmological number density of neutrinos (and antineutrinos) is given by

$$
n_{\nu}=N_{\text {eff }}\left(\frac{3}{4}\right)\left(\frac{4}{11}\right) n_{\gamma}
$$

where $n_{\gamma}$ is the density of the CMB photons, $N_{\text {eff }}$ is the effective number of neutrino species in the universe, and the factor of 3/4 comes from the difference between Fermi-Dirac and Bose-Einstein statistics. The relativistic energy density in the early universe including neutrinos is given by

$$
\rho_{R}=\left(1+N_{\mathrm{eff}} \frac{7}{8}\left(\frac{4}{11}\right)^{4 / 3}\right) \rho_{\gamma},
$$

where the factor $7 / 8$ accounts for the fact that neutrinos are fermions.

$N_{\text {eff }}$, as defined above, would be exactly equal to three if neutrinos instantaneously decoupled from the primordial plasma. However, $N_{\text {eff }}$ differs from the integer three, due to known properties of neutrinos in the early universe. First, accurate calculations show that neutrinos are still interacting with the primordial plasma when the process of electron-positron annihilation begins. Second, the energy dependence of neutrino interactions causes the high-temperature tail of the Fermi-Dirac distribution to interact more strongly, leading to an energy-dependent distortion in the energy spectrum of neutrino gas. These effects conspire to raise $N_{\text {eff }}$ to $N_{\text {eff }}=3.046$ [23]. Once the decoupling has completed, the relativistic neutrino gas redshifts while keeping its distorted energy spectrum shape ${ }^{1}$. Therefore, in the absence of non-gravitational interactions, the relativistic neutrino gas can be described by the modified effective number of neutrino species, $N_{\text {eff }}$. A high-confidence level determination of a neutrino energy density consistent with 3.046 away from the integer value would be a striking confirmation of standard cosmology, its thermal history, and convincingly show that the measured energy density is composed of Standard Model neutrinos.

Beyond Standard Model physics can also change $N_{\text {eff }}$. A particular example are additional "sterile" neutrino flavors, but in general any relativistic degrees of freedom that interact solely gravitationally (also dubbed dark radiation) will raise $N_{\text {eff }}$. A high-confidence deviation from the standard value of $N_{\text {eff }}=3.046$ would be a strong indication of new neutrino physics, new particles, or an invalidity in the theoretical assumptions going into the standard cosmological theory.

The number of neutrino species primarily affects the CMB by altering the photon diffusion (Silk damping) scale, $r_{d}$, relative to the sound horizon, $r_{s}$. The sound horizon sets the location of the acoustic peaks while photon diffusion suppresses power at small angular scales. CMB measurements are sensitive to both as angles projected over the distance to last scattering: $\theta_{s}=r_{s} / D_{A}$ and $\theta_{d}=r_{d} / D_{A}$. Sensitivity of these observables to the number of neutrino species comes from how the energy density of the neutrinos increases the expansion rate. The sound horizon scales as $1 / H$, while the diffusion scale, resulting from a stochastic process, scales instead as $\sqrt{1 / H}$. Thus, the CMB can measure the expansion rate in the early universe, and therefore the relativistic energy density, by measuring the ratio $\theta_{d} / \theta_{s}=r_{d} / r_{s} \propto \sqrt{H}$ (see [25] and references therein).

\footnotetext{
${ }^{1}$ Note that in the case of massive neutrinos, the spectrum will be altered when the neutrinos start becoming nonrelativistic and the evolution must be calculated with general relativistic kinetic theory [24], which is handled properly by modern Boltzmann solvers.
} 
Due to this effect one can infer $N_{\text {eff }}=3.36 \pm 0.34$ [1] from the combination of temperature data from Planck, WMAP polarization data [26], and high- $\ell$ CMB measurements (South Pole Telescope (SPT) [27] and Atacama Cosmology Telescope (ACT) [7]). LSS measurements improve these constraints by breaking degeneracies with other cosmological parameters. In $\S 2$, we will give the constraints from combining future CMB and LSS measurements.

\subsection{The Sum of the Neutrino Masses, $\sum m_{\nu}$}

The physical effects of massive neutrinos on the CMB and LSS have been studied for nearly two decades (e.g., [28]). When the detailed effects of the different components of matter in the universe on linear LSS were first precisely calculated (e.g., [29]), it became clear that the impact of massive neutrinos on LSS was quite large, making LSS a sensitive probe of massive neutrinos [30]. The physical effect of massive neutrinos on LSS is determined by the fact that massive neutrinos behave as radiation-like particles in the early universe, and as matter-like particles in the late universe [31, 32, 3]. Since the number density of neutrinos is comparable to that of photons (Eq. 1), they will contribute considerably to the relativistic energy density when their energies are relativistic. If the neutrinos are to affect matter clustering in a nontrivial amount, then they must have contributed a nontrivial amount to the total matter density. Because of the neutrinos' high cosmological number density, a small neutrino mass lets them contribute to the critical density as

$$
\Omega_{\nu} h^{2} \simeq \frac{\sum m_{\nu}}{93 \mathrm{eV}} .
$$

Therefore, even the minimum summed neutrino mass of $58 \mathrm{meV}$ contributes to a matter fraction $f_{\nu} \equiv \Omega_{\nu} / \Omega_{m}$ of about $0.4 \%$.

In broad terms, the neutrinos cool down by the expansion of the universe and transition from being ultrarelativistic gas behaving as radiation to being cold gas behaving as dark matter at a redshift around $z_{\mathrm{nr}} \sim 2000 m_{\nu} / 1 \mathrm{eV}$. Before they are non-relativistic, they free-stream out of over-dense regions, erasing the structure on small scales. This free-streaming establishes a preferred scale, which very roughly corresponds to the horizon scale at time of transition to the non-relativistic matter. In models with massive neutrinos, power appears suppressed on scales smaller than the free-streaming scale when normalizing the matter power spectrum at large scales. This scale numerically turns out to be close to the peak in the matter power spectrum, a feature produced because of the transition from cosmological radiation to matter domination when perturbations cross the cosmological horizon. This effect is shown in Figure 1. Measurements of this feature in cosmological LSS can be made in two ways: first through the overall change in shape of the power spectrum, or second in a relative amplitude measurement, where for example the CMB precisely measures the amplitude at large scales and a LSS probe gives a measure at small scales.

Among the most stringent current combined CMB plus LSS cosmological constraints on $\sum m_{\nu}$ result from the combination of Planck CMB data, WMAP 9-year CMB polarization data [26], and a measure of baryon acoustic oscillations (BAO) from the Baryon Oscillation Spectroscopic Survey (BOSS) [33], Sloan Digital Sky Survey (SDSS), Wigglez LSS data, and the $6 \mathrm{dF}$ galaxy redshift survey. As reported in [1], these combined probes produce an upper limit $\sum m_{\nu}<0.23 \mathrm{eV}(95 \%$ C.L.). A more aggressive use of galaxy clustering into smaller scales and the nonlinear clustering regime can lead to more stringent constraints (e.g., [34, 35]).

Sensitivity to effects of neutrinos on cosmological gravitational perturbation evolution and the matter power spectrum are measured in several ways:

Probing $\sum m_{\nu}$ with CMB temperature and polarization - The CMB constrains the neutrino mass through its effect on structure growth in two primary ways: 1) the early Integrated Sachs Wolfe (ISW) effect, 
and 2) gravitational lensing of the CMB by LSS. A significant fraction of the power in the CMB on a degree angular scale is from the early ISW effect. This measurement has been used to infer $\sum m_{\nu}<0.66 \mathrm{eV}(95 \%$ C.L.) [1]. Only marginal improvement is possible with that technique as the constraint is already limited by cosmic variance due to its reliance on the large angular scale power in the CMB.

Gravitational lensing of the CMB provides a clean and direct measurement of the matter power spectrum on scales where effects of neutrino mass manifest. Recently, ACT, SPT, and Planck have used high order statistics in temperature anisotropy to produce lensing maps. Polarization surveys will represent a large improvement over what can be done with temperature. Gravitational lensing of primordial $E$ modes by intervening matter distributions produces a $B$-mode polarization signal [36], which has recently been detected [11]. This lensing-induced $B$-mode polarization corresponds to an RMS of $\sim 5 \mu \mathrm{K}$-arcmin with a characteristic angular scale is a few arcminutes. Detailed, high signal-to-noise measurements of arcminute polarization can therefore be used to reconstruct the lensing potential. This effect has already been used to achieve modest improvements in the CMB-based neutrino mass constraints $[27,7,1]$, which we expect to improve by nearly an order of magnitude with a Stage-IV CMB experiment (see §2).

Probing $\sum m_{\nu}$ with baryonic tracers of the large scale clustering of matter - Galaxy surveys, $21 \mathrm{~cm}$ surveys, and the Lyman- $\alpha$ forest are measurements of tracers of the underlying matter clustering in LSS. It can be shown that the large scale fluctuations in the tracer will follow those of the dark matter with a scale-independent constant of proportionality as long as processes that determine the local number density of galaxies and the emission of $21 \mathrm{~cm}$ radiation among other observables are local functions of the dark matter density [37]. On smaller scales the biasing relation can become more complicated. In the case of $21 \mathrm{~cm}$ and the Lyman- $\alpha$ forest, neutral hydrogen gas is the tracer, which, to zeroth order, should follow the underlying dark matter distribution. In the case of the Lyman- $\alpha$ forest, the gas is seen via absorption features toward a distant quasar, and therefore the measure is a one-dimensional tracer. In the case of spectroscopic galaxy surveys, the tracer is that of galaxies, which have been found to have scale-independent bias at large scales, both in observations [38] and in simulations, e.g. Ref. [37].

Additional information comes from the fact that we are observing tracers in redshift-space rather than realspace, which distorts the observed two-point function in the direction along the line of sight. Since galaxies cannot experience velocity bias on very large scales, this distortion allows one to break the degeneracy between bias and the amplitude of dark matter fluctuations [39]. This further improves our ability to constrain neutrino properties by comparing the small-scale power amplitude measured by galaxy clustering and the large scale power amplitude determined by the CMB. In Fisher matrix projections described below these constraints emerge naturally by considering the anisotropic measurements of the galaxy power spectrum. Further details of this method are given in Ref. [40].

More details are given in $\S 2.2 \& 2.4$.

Probing $\sum m_{\nu}$ with weak lensing - Deep, high angular resolution observations of the sky can reveal gravitational weak lensing of background galaxies by foreground LSS, sometimes dubbed cosmic shear. The prospect of the statistical angular and tomographic correlations in the mean weak lensing signal for being a precision probe of LSS has been studied for some time [41, 42, 43]. The use of the weak lensing signature to infer the neutrino masses was first discussed via angular correlation measures in the deep nonlinear clustering regime [44] and in tomographic correlations in the linear regime [45]. The original conservative linear-regime clustering forecast of Ref. [45] determined a sensitivity of $\sum m_{\nu}<300 \mathrm{meV}$ for a 4000 square degree weak lensing survey, with a depth of one hundred galaxies per square arcminute, potentially achievable by spacebased surveys, in combination with cosmological parameter constraints from Planck CMB observations. 


\section{Forecast sensitivity to $N_{\text {eff }}$ and $\sum m_{\nu}$}

In this section, we present forecast sensitivity to cosmic neutrinos from future CMB and LSS experiments. Various complementary probes of neutrino mass are surveyed. However, we highlight a few methods considered to be least sensitive to systematic effects: an arcminute-scale CMB polarization survey from the Stage-IV CMB experiment CMB-S4 (described below and in the Appendix), galaxy clustering, and cosmic shear. The exact forecast of uncertainty depends on the cosmology and priors assumed. Our main objective in this Community Planning Study is to provide reliable comparisons between current and expected limits, and to emphasize the improvements and degeneracy-breaking capabilities of complementary probes. To meet this goal, efforts are made to consistently and systematically calculate these numbers using multiple extensively checked Fisher matrix pipelines, even though some forecast numbers disagree slightly with what is in the literature.

We warn the reader of the usual caveats associated with Fisher matrix formalism when forecasting the reach of future experiments. This formalism makes a number of simplifying assumptions including that likelihoods and posteriors are Gaussian. Some of these methods can be improved by, for example, forecasting through full likelihood evaluations using Markov Chain Monte Carlo methods and mock future data (e.g., [46, 47]). Moreover, for a lower measured mass, the posterior will have to take into account the physical limit $\sum m_{\nu}>0$, which will thus relax constraints with respect to the Fisher forecasts. In our forecasts, we use a single massive neutrino and two massless neutrinos for simplicity in not modeling the neutrino mass spectrum as well. When varying $N_{\text {eff }}$, we vary the massless neutrino density. Since the forecast sensitivity will not probe the exact neutrino mass spectrum directly, this method suffices [48].

We also note that we assume a maximum usable multipole of $\ell_{\max }=5,000$ in the analysis. (Note, here we refer to $\ell$ as CMB multipoles and $\ell^{r m W L}$ for weak lensing multipoles, below.) We find that the cosmological parameter constraints are well converged for an $\ell_{\max }$ of 4,000 , and that the range from $\ell_{\max }=3,000-4,000$ contains a small amount of (mostly polarization) information. It should be prudent to use this multipole range in our forecasts, since estimates of polarized foregrounds show these to be much smaller than the lensed primordial CMB, relatively speaking, unlike the foregrounds for temperature fluctuations (see e.g., $[49])$.

It is found in this study that for each of the probes, the expected $1 \sigma$ uncertainty on neutrino mass lies in the range of $20-30 \mathrm{meV}$. When multiple probes are combined, for example for DESI+CMB-S4, the uncertainty reduces to $\sim 15 \mathrm{meV}$. As shown in Figure 2, such a joint program will unambiguously detect neutrino mass under both hierarchy scenarios, since $\sum m_{\nu}$ is already known to be at least $58 \mathrm{meV}$.

Figure 3 shows the interplay between CMB and galaxy-survey constraints on $\sum m_{\nu}$. There is a degeneracy between neutrino mass and the matter density in the CMB lensing constraint - both parameters primarily affect the broadband amplitude of the lensing effect, with the neutrino mass also introducing a small scale dependence (see Figure 1). A low-redshift constraint on the acoustic peak scale from, e.g., DESI (see Section 2.2 for the survey description), significantly reduces this degeneracy. Because CMB lensing and galaxy clustering constrain the neutrino mass in a similar way by using some of the same information (see Figure 1), we take a conservative approach when combining CMB and galaxy surveys and only use either galaxy clustering or CMB lensing information. When CMB lensing is included, we omit clustering information in the galaxy survey and use only the BAO constraint on the acoustic scale at low redshift. When CMB lensing information is omitted, we use both $\mathrm{BAO}$ and power spectrum shape information from the galaxy surveys.

Figure 4 shows forecast constraints in the $\sum m_{\nu}-N_{\text {eff }}$ plane. Note that we do not expect that there is a physical motivation for these two parameters to be varied at the same time - we simply do this because 


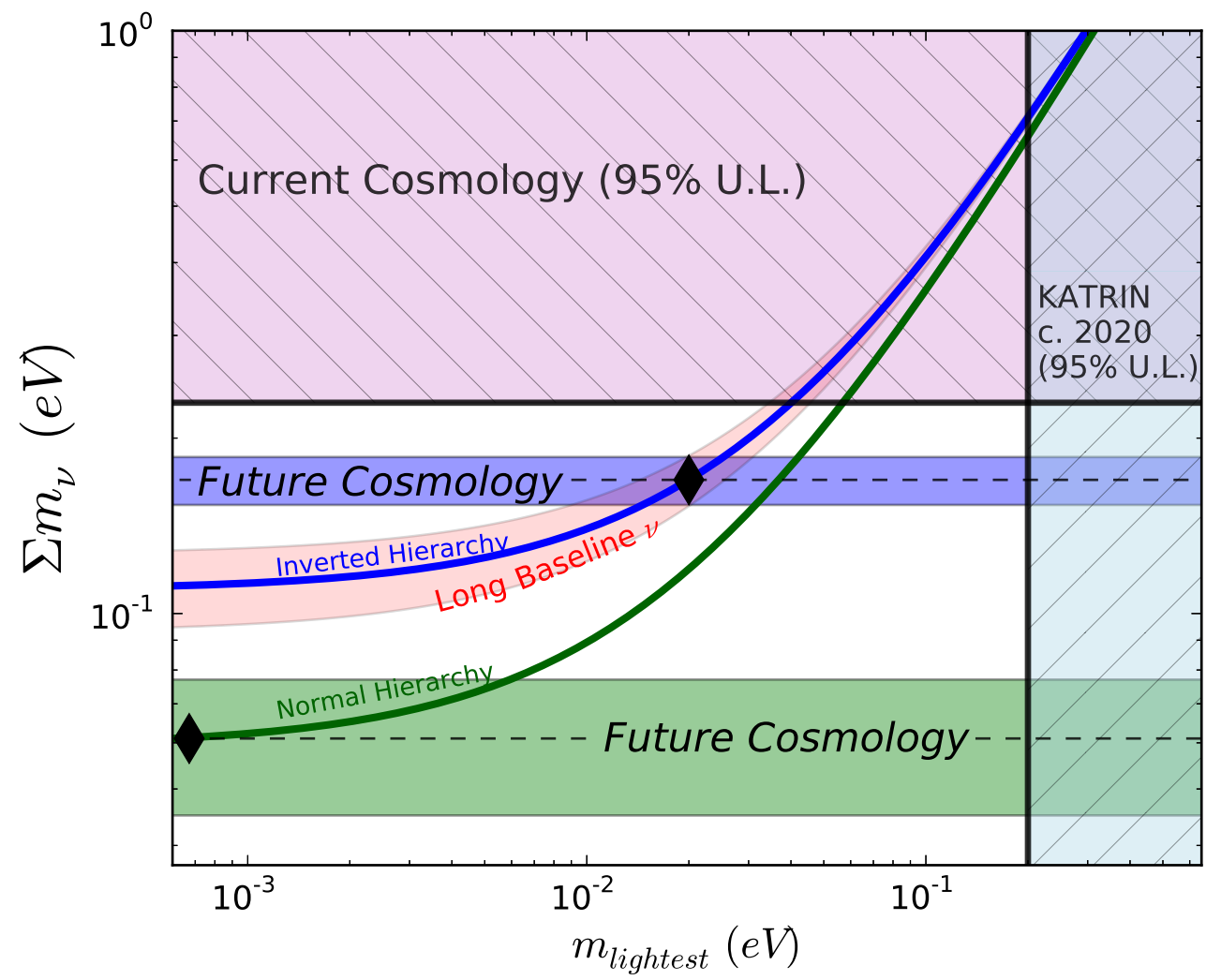

Figure 2. Shown are the current constraints and forecast sensitivity of cosmology to the neutrino mass in relation to the neutrino mass hierarchy. In the case of an "inverted hierarchy," with an example case marked as a diamond in the upper curve, future combined cosmological constraints would have a very highsignificance detection, with $1 \sigma$ error shown as a blue band. In the case of a normal neutrino mass hierarchy with an example case marked as diamond on the lower curve, future cosmology would detect the lowest $\sum m_{\nu}$ at a level of $\sim 4 \sigma$. Also shown is the sensitivity from future long baseline neutrino experiments as the pink shaded band, which should be sensitive to the neutrino hierarchy at least at $3 \sigma$ [50].

neutrino mass is an unknown quantity that needs to be marginalized over. The majority of information in this case comes from precise measurements of the photon diffusion scale relative to the sound horizon scale as described in the previous section. Here, the addition of high accuracy $E$-mode polarization measured to fine angular scales allows these two quantities to be measured with sufficient precision to decrease the error bars several-fold with respect to Planck data. The addition of broadband galaxy power spectra does not help in this case.

This accuracy will not allow us to distinguish between $N_{\text {eff }}=3$ and $N_{\text {eff }}=3.046$ at more than $2 \sigma$. However, we note that even if the true value of $N_{\text {eff }}$ is not 3.046, it is highly unlikely to be the unphysical value of a simplified model $N_{\text {eff }}=3$. We argue that the error on $N_{\text {eff }}$ is of the same order of magnitude as typical corrections stemming from detailed modeling of the thermodynamical processes in the early universe and therefore we are sensitive to the non-standard physics that would produce a signal in $N_{\text {eff }}$ order or larger than those processes. 
Table 0-1. Projections for neutrino mass and $N_{\text {eff }}$. Projections for neutrino masses are assuming a standard value of $N_{\mathrm{eff}}$. Projections for $N_{\mathrm{eff}}$ are for marginalizing over neutrino mass for galaxy clustering and CMB lensing. All values are forecasts from analyses for this work, except for the projections for galaxy weak lensing in the last two rows, which are taken from literature and referenced. All errors are $68 \%$ Fisher matrix predictions. In the case of two numbers $a / b$, these correspond to optimistic/conservative cases with $k_{\max }=0.2 \mathrm{~h} \mathrm{Mpc}^{-1}$ and $k_{\max }=0.1 \mathrm{~h} \mathrm{Mpc}^{-1}$, respectively. All values are forecasts from analyses for this work, except for the last two rows, which are referenced. Numbers that we want to highlight in this report are in bold.

\begin{tabular}{|c|c|c|}
\hline Dataset & $\sigma\left(\sum m_{\nu}\right)[\mathrm{meV}]$ & $\sigma\left(N_{\mathrm{eff}}\right)$ \\
\hline \multicolumn{3}{|l|}{ Galaxy Clustering (current CMB): } \\
\hline Planck + BOSS BAO & 100 & 0.18 \\
\hline Planck + BOSS galaxy clustering & $46 / 68$ & $0.14 / 0.17$ \\
\hline Planck + eBOSS BAO & 97 & 0.18 \\
\hline Planck + eBOSS galaxy clustering & $36 / 52$ & $0.13 / 0.16$ \\
\hline Planck + DESI BAO & 91 & 0.18 \\
\hline Planck + DESI galaxy clustering & $17 / 24$ & $0.08 / 0.12$ \\
\hline \multicolumn{3}{|l|}{ CMB Lensing (current galaxy clustering): } \\
\hline Stage-IV CMB & 45 & 0.021 \\
\hline Stage-IV CMB + BOSS BAO & 25 & 0.021 \\
\hline \multicolumn{3}{|l|}{ CMB Lensing + Galaxy clustering: } \\
\hline Stage-IV CMB + eBOSS BAO & 23 & 0.021 \\
\hline Stage-IV CMB + DESI BAO & 16 & 0.020 \\
\hline Stage-IV CMB no lensing + DESI galaxy clustering & $15 / 20$ & $0.022 / 0.024$ \\
\hline \multicolumn{3}{|l|}{ Galaxy Weak Lensing: } \\
\hline Planck + LSST [51] & 23 & 0.07 \\
\hline Planck + Euclid [48] & 25 & $\mathrm{NA}^{\dagger}$ \\
\hline
\end{tabular}




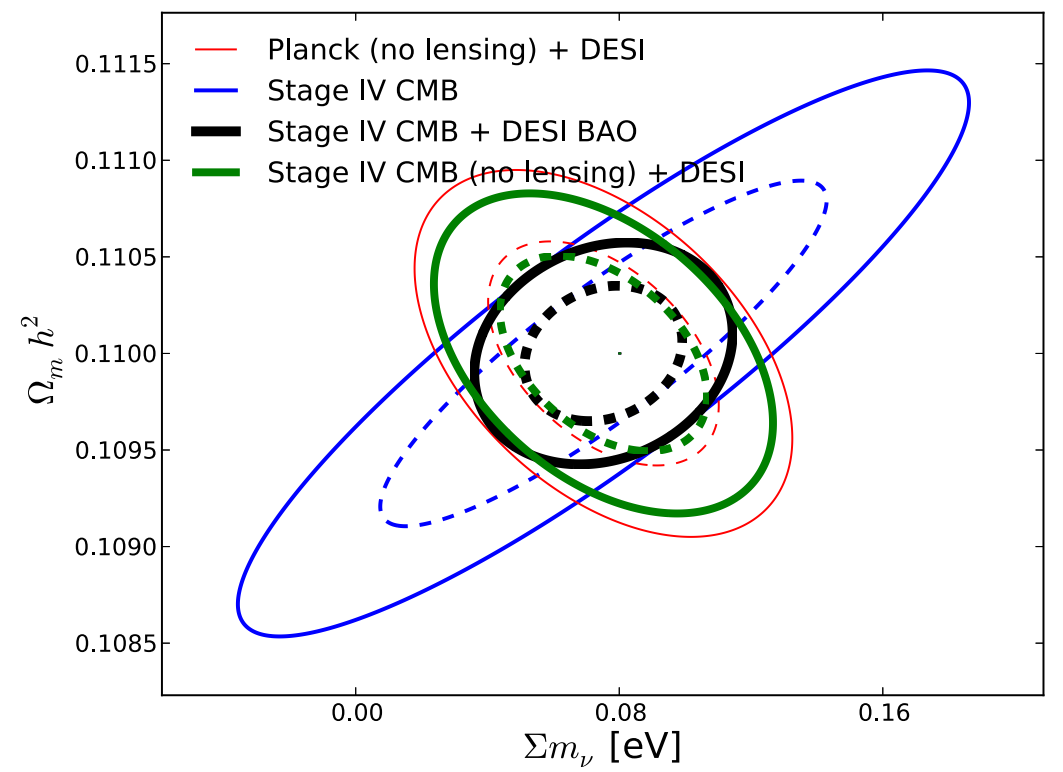

Figure 3. Forecasted $1 \sigma$ and $2 \sigma$ constraints in the $\Sigma m_{\nu}-\Omega_{m} h^{2}$ plane showing the synergy of an experiment like DESI and a Stage-IV CMB lensing experiment. For contrast, a combination of Planck data with the lensing information removed and DESI are shown in the red contours. The blue contours show the constraint generated by the CMB lensing experiment, corresponding to a $24 \mathrm{meV}$ constraint on massive neutrinos. The black contours show the result of adding only DESI BAO information, resulting in a 16 meV constraint. This can be compared to the case where no Stage-IV CMB lensing information but all galaxy clustering information is used, yielding a $24 \mathrm{meV}$ constraint. The combination of a Stage- $I V$ CMB experiment and $B A O$ information from DESI should allow a robust measurement of the sum of the neutrino masses.

\subsection{Lensing of the $\mathrm{CMB}$}

In the next decade, ground based CMB experiments will make significant progress in measuring the effects of gravitational lensing on the CMB polarization [52]. Current and upcoming CMB experiments are classified into stages with each stage corresponding to an order of magnitude increase in sensitivity, or equivalently, the number of measured modes. Stage-II experiments measure $O(1000)$ optical modes and are now providing the first statistical detections of lensing $B$-mode polarization [11]. Many of the expected properties of the lensing signal, such as its amplitude and statistics, will be measured with real data for the first time in the Stage-II era. Stage-III experiments will deploy in the latter half of the decade and measure $O(10,000)$ modes. These experiments will be the first instruments with sufficient sensitivity to make high signal-to-noise maps of the CMB lensing modes over a few thousands square degrees of sky. Stage III will correspond to a transition of the CMB lensing measurement from a statistical detection into imaging. A Stage-IV experiment observing $O(100,000)$ modes is anticipated to deploy in $\sim 2020$. Stage-IV experiments will build on Stage III to map tens-of-thousands of square degrees of sky to a depth of $\leq 1 \mu \mathrm{K}$ per 1 -arcminute pixel with an angular resolution of $\leq 3$ arcminutes. In the Appendix, we describe the technical program for CMB-S4, a Stage-IV CMB experiment which aims to deploy in 2020 and operate for several years. One of the most exciting prospects of a Stage-IV experiment like CMB-S4 is to measure the signatures of cosmic neutrinos with high precision. 


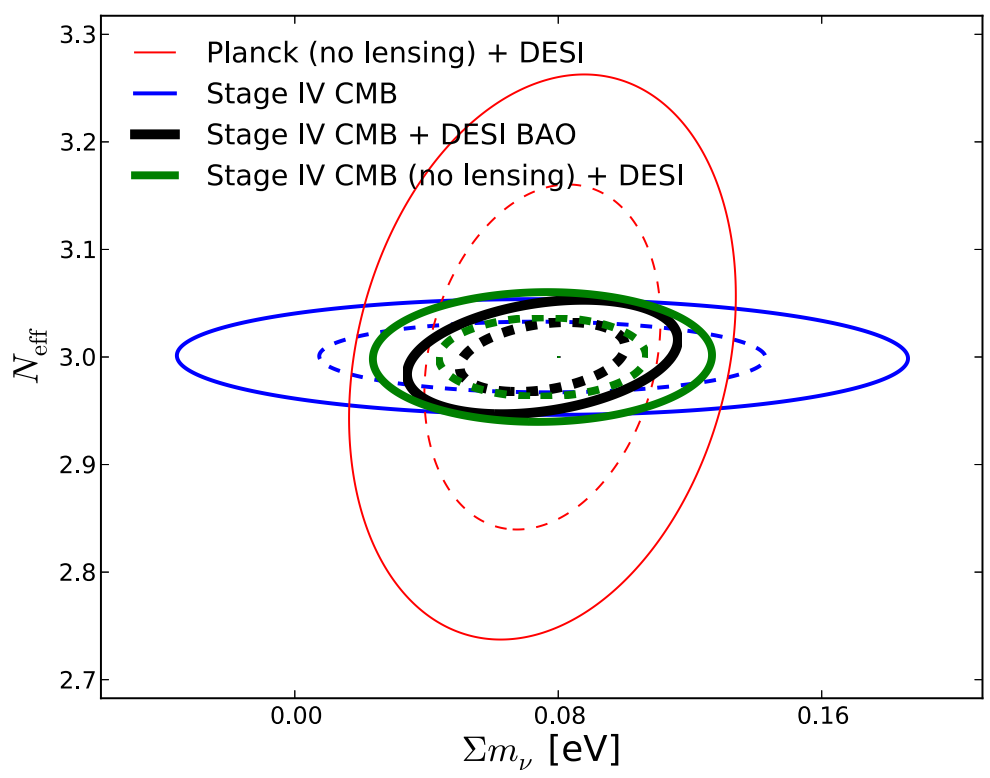

Figure 4. The same as Figure 3, but showing forecasts in the $\Sigma m_{\nu}-N_{\text {eff }}$ plane for a model including the effective number of neutrino species as a free parameter. A Stage-IV CMB experiment will not be able to distinguish between the standard model value of $N_{\mathrm{eff}}=3.046$ and the integer value of 3 at high statistical significance, but it will indicate a preference for one over the other at the $\sim 2 \sigma$ level.

CMB polarization as a probe of large scale structures has a few unique advantages. First of all, CMB lensing is highly complementary to galaxy surveys, since it probes matter distributions in the linear regime at higher redshift $(z \sim 2-4)$. Secondly, because the unlensed background is precisely understood (Gaussiandistributed E-mode polarization at redshift $z=1090$ in the absence of non-Gaussianities, which are strongly limited in the primordial CMB [53]), the reconstruction of lensing potential is absolutely calibrated and free of shape noise. This property also enables reconstruction beyond the quadratic order, with sensitivity only limited by instrumental noise. Finally, the systematics associated with CMB lensing originated largely from well-understood instrumental effects, which tend to decrease with higher resolution.

Figure 5 shows the projected constraints on the CMB lensing potential power spectrum $C_{L}^{\Phi \Phi}$ for a Stage-IV CMB experiment, along with the fractional change in $C_{L}^{\Phi \Phi}$ for some fiducial values of $\sum m_{\nu}$ relative to the $\sum m_{\nu}=0$ case.

\subsection{Tomographic galaxy clustering with spectroscopic surveys}

Starting in 2014, the Extended Baryon Oscillation Spectroscopic Survey (eBOSS) will use the BOSS spectrograph to perform spectroscopy on a massive sample of galaxies and quasars in the redshift range that lies between the BOSS galaxy sample and the BOSS Lyman- $\alpha$ sample. The targets for eBOSS spectroscopy will consist of Luminous Red Galaxies (LRGs: $0.6<z<0.8$ ), Emission Line Galaxies (ELGs: $0.6<z<1.0$ ), "clustering" quasars to directly trace large-scale structure $(1<z<2.2)$, and Lyman- $\alpha$ quasars $(2.2<z<$ 


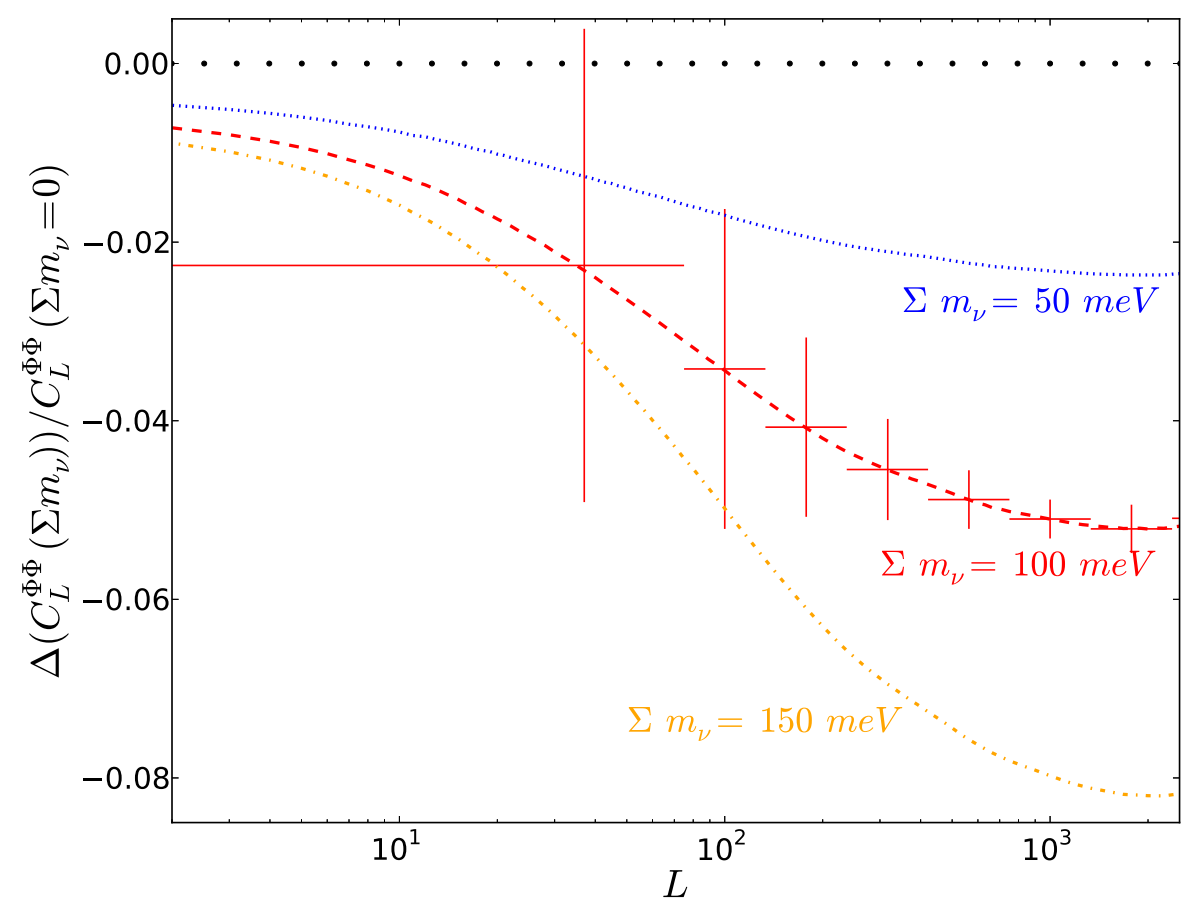

Figure 5. The effect of massive neutrinos on the CMB lensing potential power spectrum $C_{L}^{\Phi \Phi}$. The fractional change in $C_{L}^{\Phi \Phi}$ for a given value of $\sum m_{\nu}$ is shown relative to the case for zero neutrino mass. Projected constraints on $C_{L}^{\Phi \Phi}$ for a Stage- $I V$ CMB experiment are shown for $\sum m_{\nu}=100 \mathrm{meV}$. Here we have approximated all of the neutrino mass to be in one mass eigenstate and fixed the total matter density $\Omega_{m} h^{2}$ and $H_{0}$. The $1 \sigma$ constraint for $\sum m_{\nu}$ is approximately $45 \mathrm{meV}$ for lensing alone and drops to $16 \mathrm{meV}$ when combined with other probes.

3.5). The comoving volume probed by eBOSS will be nearly ten times that probed by the BOSS galaxy survey (though at lower sampling density).

The success of BOSS not only led to the eBOSS program, but has largely inspired the concept of DESI. DESI is a proposed wide-field spectroscopic survey, to be conducted on a 4-m class telescope to study dark energy. DESI represents one of the top priorities for new Office of Science Cosmic Frontiers efforts as identified in the recent "Rocky III" report; CD-0 approval was granted in September 2012. The preliminary DESI survey design covers 14,000 deg ${ }^{2}$ with spectroscopic observations of LRGs $(0.6<z<1.0)$, ELGs $(0.6<z<1.5)$, and quasars $(1<z<3.5)$ for direct clustering and Lyman- $\alpha$ forest. The density and effective volume probed by DESI will far exceed either BOSS or eBOSS. The details of the survey specifications are provided in Ref. [40].

As a natural consequence of large spectroscopic BAO programs such as BOSS, eBOSS, and DESI, clustering in the density field is sampled across a wide range of $k$ modes over a very large volume. As explained in Section 1.3, the signature of neutrinos appears as a characteristic suppression of power below certain scales in the matter power spectrum. Effort within the BOSS collaboration has primarily centered on reconstructing the broad-band power spectrum to extract information about BAO, redshift space distortions, and bias 
parameters from galaxies and Lyman- $\alpha$ forest. Continued improvements on estimates of the power spectrum will soon allow tests of neutrino masses with the BOSS data.

Assuming a baseline of Planck CMB measurements, we forecast for sensitivity for neutrino mass with BOSS, eBOSS and DESI [40]. We assume measurements of large-scale modes with wavelengths up to $k_{\max }=$ $0.1 \mathrm{~h} \mathrm{Mpc}^{-1}$ (conservative estimates) and $k_{\max }=0.2 h \mathrm{Mpc}^{-1}$ (optimistic estimates). ${ }^{2}$ Results can be found in the Table $0-1$.

For the eBOSS experiment, we predict a $68 \%$ measurement error of $\sigma\left(\sum m_{\nu}\right)=36 / 52 \mathrm{meV}$ for optimistic/conservative estimates when combining the results from the eBOSS LRGs, ELGs and quasars. The upper limit on neutrino masses derived from eBOSS is comparable to the minimum allowed mass in an inverted hierarchy. Current constraints on the effective number of neutrino species of $N_{\text {eff }}=3.30 \pm 0.27$ from BAO and Planck CMB data will be improved by a factor of two by including eBOSS measurements. DESI should improve these constraints considerably. In particular, neutrino masses can be constrained to $\sigma\left(\sum m_{\nu}\right)=17 / 24 \mathrm{meV}$ (optimistic/conservative) from galaxies alone, enough to make a measurement of the minimum neutrino mass at $\sim 3 \sigma$ confidence.

\subsection{Tomographic Weak Lensing in Galaxy Surveys}

Recent determinations of the sensitivity of upcoming ground based surveys such as the Large Synoptic Survey Telescope (LSST) [54] have a forecast sensitivity of deep background galaxy imaging at the level of $\sigma\left(\sum m_{\nu}\right)=23 \mathrm{meV}$ [51]. This value includes the tomographic galaxy clustering information that would also be achieved from LSST covering one-half of the sky, an average galaxy density of $50 \mathrm{arcmin}^{-2}$, using methods discussed in $\S 2.2$. A space-based mission with wide field of view and covering $10 \%$ of the sky with $100 \operatorname{arcmin}^{-2}$ sensitivity and mean lensed galaxy redshift of $\langle z\rangle=1$ was found to have the sensitivity of $\sigma\left(\sum m_{\nu}\right)=23 \mathrm{meV}$ as well. Such a survey may be achieved by some configurations considered for the proposed Wide-Field Infrared Survey Telescope-Astrophysics Focused Telescope Asset (WFIRSTAFTA) [55]. Both of these forecasts employed simultaneous Planck-level determinations of cosmological parameters, but allowed for extra parameter variation, including $N_{\text {eff }}$ and running of the primordial power spectrum index [51]. Relaxing from a non-minimal cosmological model, as discussed in $\S 3.1$ relaxes these constraints.

The upcoming European Space Agency EuCLID mission will also perform a deep galaxy survey and weak lensing survey [48]. Planck CMB plus EuCLID weak lensing has been forecast to have a sensitivity of $\sigma\left(\sum m_{\nu}\right)=25 \mathrm{meV}$ in a minimal cosmological model, with the upper limit depending on assumptions on galaxy bias [56, 57]. Forecasts of a combination of angular and tomographic weak lensing shear autocorrelation, galaxy auto-correlation, and galaxy-shear cross-correlation sample data from EucLID has been forecast to have a sensitivity of $\sigma\left(\sum m_{\nu}\right)=23 \mathrm{meV}$, when including a marginalization over the galaxy bias model [57]. The photometric galaxy cluster catalog would also have sensitivity to neutrino mass [58]. It has been found in Ref. [59] that $\sigma\left(\sum m_{\nu}\right)$ could improve to $10 \mathrm{meV}$ range with the full combination of weak lensing, galaxy clustering, galaxy-shear cross-correlation, in combination with a new a weak-lensing selected galaxy cluster sample derived from the EuCLID galaxy survey. However, this sensitivity would require large improvements in our understanding of observational systematics of all of these combinations of probes.

\footnotetext{
${ }^{2}$ These constraints are somewhat more conservative that those used in [1], where $k_{\max }$ of $0.15 h \mathrm{Mpc}^{-1}$ and $0.3 h \mathrm{Mpc}^{-1}$ were used as conservative and optimistic estimates respectively.
} 


\subsection{Other Probes: Galaxy cluster surveys, Lyman- $\alpha$ Forest, and $21 \mathrm{~cm}$ Surveys}

There have been forecasts for a number of other tests of cosmological LSS as a probe of neutrino masses. These include the clustering of neutral gas as probed in the Lyman- $\alpha$ forest, galaxy cluster samples, and $21 \mathrm{~cm}$ surveys. These methods also have complicated relations between the observables and inferred linear primordial LSS clustering, yet the inferred sensitivity on neutrino mass from these observations may be competitive with other probes.

The Lyman- $\alpha$ forest is the pattern of absorption features in quasar spectra due to intervening low density gas. This gas is expected to follow gravitationally-dominant dark matter clustering in the linear to weakly non-linear regime of clustering. Going from the observed flux distribution to the power spectrum of matter in LSS requires knowing the bias of gas to matter, which includes knowing the temperature-density relation of the gas and its evolution over cosmic history, as well as the nature of the ionizing background radiation impinging on the gas. Setting aside the systematic uncertainties, the Lyman- $\alpha$ forest has some of the strongest inferred constraints on neutrino mass: using results from WMAP 3-year CMB observations with SDSS quasar's Lyman- $\alpha$ forest, the upper limit on the neutrino mass is $\sum m_{\nu}<0.17 \mathrm{eV}$ (95\% C.L.) [60]. We note however, that the calibration of the absolute power amplitude in the subsequent WMAP releases has changed in the direction that would relax these limits. The Planck absolute power measurements are even higher that the final WMAP measurements, further weakening these constraints. Follow-up analyses found that it is possible to constrain neutrinos even in the absence of CMB data prior giving an upper limit of $\sum m_{\nu}<0.9 \mathrm{eV}(95 \%)$ based on SDSS quasar's Lyman- $\alpha$ forest [61]. Optimistic forecasts for highresolution Keck and/or VLT spectra of the Lyman- $\alpha$ forest combined with Planck-level CMB constraints find their reach to be $\sum m_{\nu}<0.11 \mathrm{eV}$ (95\% C.L.) [62]. Modeling of the Lyman- $\alpha$ forest through numerical simulations is a very active field with several U.S. and European groups currently doing research. It is likely that the future constraints will be limited by the systematic accuracy with which we can make these theoretical predictions.

An upcoming probe of the high-redshift gas is low-frequency radio observations of the redshifted $21 \mathrm{~cm}$ line of neutral hydrogen, which has the potential to map the matter distribution through the epoch of reionization, out to $z \sim 12$. A key requirement of $21 \mathrm{~cm}$ surveys is the removal of Galactic synchrotron emission, which is four to five orders of magnitude greater than the $21 \mathrm{~cm}$ signal from high- $z$. The smoothness of the foreground has been shown to be usable to overcome its dominance and $21 \mathrm{~cm}$ clustering measurements out to $z \sim 0.8$ have been achieved [63]. Because of the potential of mapping neutral hydrogen to high-redshifts, large volumes of matter clustering may be measurable, revealing an unprecedented number of $k$ modes [64]. Several $21 \mathrm{~cm}$ experiments are operational or being developed, including the LOw Frequency ARray (LOFAR ${ }^{3}$, the Precision Array for Probing the Epoch of Reionization (PAPER) ${ }^{4}$, the Murchison Widefield Array (MWA) ${ }^{5}$, and the Canadian Hydrogen Intensity Mapping Experiment $(\mathrm{CHIME})^{6}$. The highest sensitivities would come from a large-scale $21 \mathrm{~cm}$ survey facility proposed as the Square Kilometer Array (SKA) ${ }^{7}$. For example, forecasts for MWA sensitivities to neutrino mass, with Planck CMB constraints, are $\sigma\left(\sum m_{\nu}\right)=27 \mathrm{meV}$, and for SKA plus Planck reach $\sigma\left(\sum m_{\nu}\right)=17 \mathrm{meV}$ in optimistic scenarios of ionization modeling, but can decrease in sensitivity by a factor of $\sim 3$ or more for less optimistic modeling scenarios [65].

Galaxy clusters are the most massive bound structures in the universe, and their abundance is highly sensitive to the amplitude and shape of the matter power spectrum on scales of $k \approx 0.1 \mathrm{Mpc}^{-1} h$. Galaxy cluster samples have been created from X-ray surveys detecting intra-cluster gas emission, optical surveys of

\footnotetext{
${ }^{3}$ http://www. lofar.org/

${ }^{4}$ http://astro.berkeley.edu/ d dbacker/eor/

${ }^{5}$ http://www.mwatelescope.org/

${ }^{6}$ http://www.physics.ubc.ca/chime/

${ }^{7}$ http://www. skatelescope.org
} 
member galaxy clusters, and millimeter-wave surveys which detect the Sunyaev-Zeldovich (SZ) effect from inverse Compton scattering. A limiting systematic in galaxy-cluster based measures of the primordial power spectrum and therefore neutrino mass is the connection between the observable quantity and the inferred galaxy cluster halo mass, the latter of which is predicted by structure formation simulations. Forecasts for weak-lensing-selected galaxy clusters from an LSST-like surveys are optimistic with sensitivities of $\sigma\left(\sum m_{\nu}\right)=30 \mathrm{meV}[66]$. Optically-selected galaxy cluster samples can improve on the constraints from galaxy-clustering and weak-lensing measures, as discussed in $\S 2.3$.

\section{Systematics}

\subsection{Theoretical Priors}

The goal of constraining and potentially detecting neutrino mass and neutrino number density cosmologically is based within a rigorously constrained and well-tested model. However, that model has some inherent theoretical priors and simplifying assumptions. Almost all of the constraints on neutrino mass discussed here and in the literature are constraints on neutrinos as an extension to the standard cosmological model, minimally described by six parameters, but sometimes extended to up to 10 parameters. A detailed discussion of the model dependence of the cosmological constraints on neutrino mass was given in Ref. [67], and we summarize some of the model dependencies here. As discussed in the introduction, if a non-minimal model is detected robustly by cosmological observations, with $N_{\text {eff }} \neq 3.046$ and $\sum m_{\nu} \gg 58 \mathrm{meV}$, this opens the possibility of an indication for other extensions to cosmology, including curvature, non-constant dark energy, a non-uniformly scale invariant primordial perturbation spectrum, extra particle or radiation species, and even other possibilities. Conversely, if the minimal model is detected with high significance, that detection rests on the theoretical edifice of modern cosmology, albeit a successful model. If laboratory experiments of neutrino mass and properties are at odds with the cosmological results, then the implications for cosmological theory would necessarily be addressed.

Other theoretical assumptions are also present even within the 10-parameter models that are not commonly explicitly stated. These include the assumption that the primordial power spectrum arising out of inflation is a power-law, $P(k) \propto k^{n}$, or, in extended models at most having a curvature "rolling" of the power law, $P(k) \propto k^{n+(d n / d \ln k)\left(k / k_{0}\right)}$. There has been some work that shows that dropping the assumption of a nearly scale-independent power law spectrum could provide cosmological observations that have drastically different neutrino sectors, but produce the same CMB observations [68]. Other work has shown that constraints on cosmological parameters expand considerably when including arbitrary yet continuous functions for the initial conditions of the primordial perturbation spectrum [69]. That work did not address neutrino constraints specifically, but presumably neutrino mass constraints would also greatly degrade once the assumption of the primordial spectrum is relaxed similarly. It should be emphasized that deviations from a primordial nearly scale-invariant spectrum are not predicted by standard inflation models [70]. Other implicit assumptions include that Einstein's gravity is valid on galaxy to cosmological scales (e.g., [71]).

Note that there also exist model-dependent assumptions in some of the LSS observations' interpretations leading to the constraints described in the previous sections. These include the nature of galaxy biasing with respect to dark matter clustering in the galaxy auto and cross-correlation statistics studied, e.g, in Ref. [59]. In either optically selected or weak lensing selected cluster-counting constraints, the mass function of halos and the mass-observable relation have inherent model uncertainties that are only partially quantified, at this point. In addition, some of the high angular resolution weak lensing shear constraints use information from where weak lensing has the highest signal-to-noise, at weak-lensing $\ell^{\mathrm{WL}}$ multipoles where matter clustering 
is in the deeply nonlinear regime. This would require an accurate and precise prediction of the nonlinear matter power spectrum [72] including significant effects on matter clustering from baryons [73, 74, 75] and even neutrino clustering [76, 77, 78, 79, 80].

\subsection{CMB Foregrounds and Systematics}

Reconstruction of the matter deflection field from observations of the lensed CMB - and, by extension, CMB lensing constraints on neutrino physics - rely on the non-Gaussian nature of the lensed CMB; the deflection of $\mathrm{CMB}$ photon trajectories by the intervening gravitational potential induces correlations between initially independent spatial modes, and this permits the potential to be reconstructed. Because astrophysical foregrounds are non-Gaussian they can affect lensing reconstruction in a complicated way. Similarly complicated will be the way in which instrumental polarization systematics come into the higherorder calculations involved in lensing reconstruction. Fully quantifying these effects will be an involved task that will almost certainly require end-to-end simulations.

For the purpose of this Community Study, we assess the effects of foregrounds and systematics on lensing reconstruction in a simplified way. Since $B$-mode polarization is by far the faintest signal used in lensing estimators, the foregrounds and systematic errors in lensing reconstruction have the largest impact on measurements of $B$-mode polarization. If we simply ensure that the false $B$ modes from astronomical and systematic residuals are smaller than the noise level, their effects on the much larger $E$-mode signal will be completely negligible, and lensing reconstruction will be noise-limited. This significantly simplifies the considerations of frequency coverage and instrument specifications, because predicting the levels of astronomical and instrumental $B$-mode polarization is much more straightforward than dealing with higherorder statistics. In addition, a large volume of literature already exists on the forecast and mitigation techniques, thanks to the global pursuit of tensor-mode perturbations (e.g., $[81,82,83]$ ). $B$-modes from primordial gravitational waves are at larger scales, smaller $\ell$ 's, and do not largely affect the lensing $B$-mode reconstruction of the scales of interest [52].

\subsubsection{CMB Foregrounds: Polarized Point Sources}

Focusing on the level of residuals in $B$-mode measurements, we can quickly conclude that polarized extragalactic point sources are expected to be the largest contaminant of the reconstructed potential [49]. The $B$-mode power spectrum from point sources is dominated by bright sources, which can be identified and masked up to a flux limit. The level of residual $B$ modes after source cuts depends on the degree of polarization of the sources. The mm-wave flux density distributions of both radio sources and dusty galaxies have been well established in recent years $[84,85,86]$. The polarization properties of these sources at $\mathrm{mm}$ wavelengths is less well-known, but strong upper limits can be placed on the mean-squared polarization fraction of both source families [87, 88]. Using these upper limits as maximally pessimistic assumptions for foreground contamination, robust predictions can be made for CMB-S4. In the $2500 \mathrm{deg}^{2}$ SPT-SZ survey, with a $\sim 6 \mathrm{mJy}$ cut, the residual source rms is already below $10 \mu \mathrm{K}$-arcmin in temperature. Since the expected source polarization is $2-5 \%$, the residual $\mathrm{rms}$ in polarization will be significantly below $1 \mu \mathrm{K}$-arcmin, the benchmark sensitivity for CMB-S4. At $95 \mathrm{GHz}$ and $150 \mathrm{GHz}$, there is approximately one source per square degree with a flux density greater than $6 \mathrm{mJy}$. Therefore, they can be easily identified and removed with minimal data loss using internal CMB-S4 data (a 6 mJy source will be detected at $\sim 30 \sigma$ for a $95 \mathrm{GHz}$ experiment with $\sim 1 \mu \mathrm{K}$-arcmin and $3^{\prime}$ FWHM). 


\subsubsection{CMB Instrumental Systematics}

Tight control of all potential sources of systematic contamination is required for any deep measurement of faint $B$-mode polarization signals. Since lensing reconstruction is ultimately limited by the amount of false $B$-mode signal, the methodology developed for predicting and mitigating instrumental systematics for inflationary $B$ modes can be directly used to estimate the experimental specifications for CMB lensing. Beam imperfections, gain uncertainties, and polarized sidelobe pickup can all convert much brighter temperature fluctuations to false $B$ modes. Errors in polarization angle calibration or telescope pointing can falsely mix brighter $E$ modes into $B$.

Drawing on the extensive literature on polarization systematics, we conclude that the most potentially dangerous instrumental sources of spurious $B$-mode polarization are gain and beam errors that mix $T$ into $B$. For example, a gain mismatch between detectors measuring two orthogonal polarizations directly converts $T$ anisotropy to polarization ("monopole leakage"). The required level of gain control for lensing science can be estimated by simply requiring the $T$ leakage be smaller than the noise level in $B$. For CMB-S4 noise levels ( $\sim 1 \mu \mathrm{K}$-arcmin), we estimate that if gain mismatch is controlled at the $<10^{-3}$ level, the false $B$ signal will be less than the noise level for $\ell>300$, where most of the lensing information resides. This level of relative gain calibration is challenging, but the requirement can be significantly alleviated by projecting measured $T$ modes out of polarization maps. This technique removes any bias from $T \rightarrow B$ monopole leakage, at the cost of a slight increase in variance. The required level of relative gain calibration is relaxed to $10^{-3} \xi$, where $\xi$ is the signal-to-noise ratio of the $T$ template. Since $\xi$ is easily in the hundreds with CMB-S4 $T$ maps, the required calibration precision will become very manageable.

A beam mismatch between detectors measuring orthogonal polarizations also converts $T$ anisotropy to a false polarization signal, but with some dependence on angular scale (as opposed to the monopole leakage from gain mismatch). Because the dominant modes of this systematic are a dipole and quadrupole at the scale of the beam, the effect strongly mitigated when the instrument beam size is smaller than the smallest scientific scale of interest. For example, a $3^{\prime}$ FWHM beam places the peak of the quadrupole beam-mismatch effect at $\ell \sim 15,000$, an order of magnitude above the peak of the lensing $B$-mode spectrum.

\subsection{Deviations from Uniformity of Density Tracers in LSS}

The Fisher matrix projections for neutrino mass constraints from spectroscopic LSS surveys assume that the large-scale clustering measurements will be limited by statistical errors. This requires stringent control of systematic errors such as incomplete modeling of astrophysical effects and systematic errors arising from instrument performance and survey design.

The astrophysical systematics arising from scale-dependent biasing are perhaps not such a big problem as one would naively expect, since neutrino suppression creates a very specific distortion of the matter power spectrum. At the scales of interest, other effects will receive perturbative corrections in even powers of $\left(k / k_{x}\right)$, where $k_{x}$ is the relevant small scale (non-linear scale for non-linear clustering, locality scale for biasing, etc.), using methods for non-linear modeling as discussed, e.g., in Refs. [89, 90]

On the other hand, experience with BOSS has found that observational systematics can be significant. Contamination arises because intrinsic fluctuations on the largest scales are small and systematics effects can modulate the data on arbitrarily large scales. Examples of such contamination include the impact of stellar contamination and dust extinction on target selection efficiency, variations in seeing during imaging 
that alter target selection, varying atmospheric conditions in spectroscopy that effect redshift success, and so on.

$\mathrm{BAO}$ measurements are largely protected from such effects on data quality because they rely on a relatively sharp feature, but constraints on neutrino masses require a well calibrated measure of absolute power as a function of wavenumber. These effects are therefore important primarily for neutrino mass estimates and constraints on the effective number of neutrino species because they use the full shape of the galaxy power spectrum.

These systematics have already been extensively studied within BOSS [91, 92, 93, 94], and the greater volume and greater statistical power at large scales from eBOSS will place new demands on homogeneity of the target samples. At this point in time, the eBOSS target selection is being developed and tested for systematic effects arising from non-uniform imaging data. The primary tests conducted to date include measures of the angular correlation function and assessment of uniformity as a function of imaging data quality. In addition, more advanced surveys such as eBOSS will utilize mixed tracers of the density field in overlapping regions, allowing cross-correlation analysis as a probe of systematic trends in any single target class. Just as the lessons from BOSS have informed eBOSS target selection, eBOSS observations will be an essential step in preparation of target selection and survey design for DESI.

\section{Conclusions}

The experimental quantification of the cosmological neutrino background has achieved the robust detection of the neutrino background energy density through its effects on the CMB and LSS, and the measures of properties of neutrinos in the cosmological background have achieved unprecedented precision and accuracy. These measures compete in precision with laboratory probes of neutrino number and mass, albeit with the theoretical priors discussed in $\S 3.1$. The current generation of Planck CMB with high- $\ell$ CMB, SDSS, BOSS and 6dF LSS data have constrained the sum of neutrino masses to be $\sum m_{\nu}<0.23 \mathrm{eV}$ (95\% C.L.) and number of neutrinos to be $N_{\text {eff }}=3.30 \pm 0.27$.

We have reviewed the planned and proposed future experiments that would be more sensitive to cosmological neutrinos, including galaxy surveys, weak lensing surveys, other LSS observations, as well as a Stage-IV CMB experiment. We find that the experiments individually achieve optimal sensitivities to the sum of neutrino masses at the level of $\sim 20-30 \mathrm{meV}$, while combined CMB plus LSS probes achieve greater sensitivities.

Much of the sensitivity to $\sum m_{\nu}$ comes from measurements of the gravitational lensing of the CMB and from measurements of galaxy clustering. Significant progress has been made recently in both of these areas, with the first projected mass reconstructions from CMB lensing [5, 6, 7, 8, 9, 10], detection of the CMB lensing $B$-mode polarization [11], and percent level measurements of the distance scale from BAO measurements $[12,13,14,15]$.

We find that future combined probes of a Stage-IV CMB experiment with BAO information from the MSDESI galaxy survey can achieve

$$
\begin{aligned}
& \sigma\left(\sum m_{\nu}\right)=16 \mathrm{meV}, \\
& \sigma\left(N_{\text {eff }}\right)=0.020,
\end{aligned}
$$

in the case of a standard six parameter $\Lambda \mathrm{CDM}$ model extended by the respective neutrino parameter.

Discrimination between changes to neutrino properties and other changes to the standard cosmological model will be supported by the diversity of very specific observable differences generated by changes to neutrino 
properties. For example, increasing $N_{\text {eff }}$ not only suppresses small-scale fluctuation power, but also leads to detectable change to the temporal phase of the acoustic oscillations. Increasing $\sum m_{\nu}$ in a manner that is consistent with the CMB power spectrum leads to very specific redshift-dependent changes to the expansion rate that change sign at $z \sim 1$, as well as specific scale and redshift-dependent changes to the power spectrum of the galaxy distribution. In short, neutrinos leave a very specific imprint on various observables and these signatures will provide an important battery of consistency checks.

Given that the minimal normal-hierarchy model of neutrino mass requires a sum of neutrinos of at least 58 $\mathrm{meV}$, and a standard thermal history requires $N_{\text {eff }}=3.046$, then experimental and observational neutrino cosmology can move beyond a detection of the neutrino background, where we are today, to a precise detection of the "fingerprints" of both neutrino mass and number in the standard cosmological model. Complementarily, a robust detection away from the standard predictions would be a definitive detection of new physics. 


\section{Appendix: A Stage-IV CMB experiment, CMB-S4}

CMB-S4 is a Stage-IV CMB experiment with two goals: to search for inflationary B-modes and to measure the sum of neutrino masses to an accuracy of $10-15 \mathrm{meV}$ at $68 \%$ confidence. It occupies a unique position in the HEP science portfolio as the measurement of CMB polarization is the only method known to probe high-scale physics. As discussed in this document, measurement of CMB polarization provides a powerful and complementary approach to understanding the neutrino masses. The Stage IV CMB-S4 experiment is the logical next step for the U.S. world-leading ground-based CMB program. It will provide the order of magnitude increase in sensitivity required to reach the goal of $\sigma\left(\Sigma m_{\nu}\right)=16 \mathrm{meV}$ (when combined with data from DESI) and an uncertainty in the tensor to scalar ratio of $\sigma(r)=0.001$.

To achieve these goals, CMB-S4 will observe $50 \%$ of the sky to the unprecedented noise level of $\leq 1 \mu \mathrm{K}$ per arcminute pixel with an angular resolution of $\leq 3$ arcminutes. At higher angular resolutions the sensitivity of CMB-S4 to secondary CMB anisotropy would greatly expand its science reach, e.g., through probing Dark Energy and $\Sigma m_{\nu}$ through Sunyaev-Zel'dovich (SZ) cluster cosmology. Reaching noise levels of $\leq 1 \mu \mathrm{K}-$ arcminute is an ambitious undertaking requiring of order 500,000 background limited detectors on the sky with broad spectral coverage over $40-240 \mathrm{GHz}$, and integrating for several years. The detectors would be distributed across multiple platforms at two or more sites to provide large sky coverage and optimized optical throughput. Thus, realizing CMB-S4 requires reshaping the HEP CMB program from its current state of fragmented small efforts into a coherent well-supported experimental program.

\section{Detector Technology for CMB-S4}

The technical requirements for CMB-S4 are dictated by the fundamental limitations of CMB measurements. Specifically, all competitive CMB detectors are sensitivity-limited where the dominant noise in an individual detector element comes from shot noise arising from the arrival time of the photons. Thus, achieving the required CMB-S4 sensitivity requires increasing the number of detected modes, which is straightforward to achieve by increasing the number of detectors (see Fig. 6). CMB-S4 will have 500 times more detectors than the current state-of-the-art Stage II experiments, 30 times more than planned Stage III experiments, making scaling the primary technical challenge of CMB-S4.

Towards this end, CMB-S4 will utilize Transition Edge Sensor (TES) bolometers as its baseline detector technology. ${ }^{8}$ A TES is an ultra-sensitive thermometer consisting of a thin superconducting film weakly heat-sunk to a bath temperature much lower than the superconductor $T_{c}$ (see Fig. 7, left). The principles of operation are simple to understand. By supplying electrical power to the TES, we can raise the temperature of the sensor so that the film is in the middle of its superconducting-to-normal transition (see Fig. 7, right). If the electrical power is supplied via a voltage bias, a negative feedback loop is established [95]. Small changes to the TES temperature, arising from thermal fluctuations (noise) or changes in the absorbed power from a source (signal), lead to large changes in the TES resistance. The change in resistance creates a canceling effect because increases (or decreases) in temperature produce decreases (or increases) in Joule heating power. This negative electro-thermal feedback is very strong because the transition is very sharp. It linearizes the detector response and expands the detector bandwidth.

The TES has a number of strengths making it the best technology to pursue for CMB-S4. First, TES detectors are fabricated via micro-machining of thin films deposited on silicon wafer substrates. As a

\footnotetext{
${ }^{8}$ We note that at the lowest frequencies envisioned, $\sim 40 \mathrm{GHz}$, MMIC-amplifier or new superconducting amplifier technologies may be practical.
} 


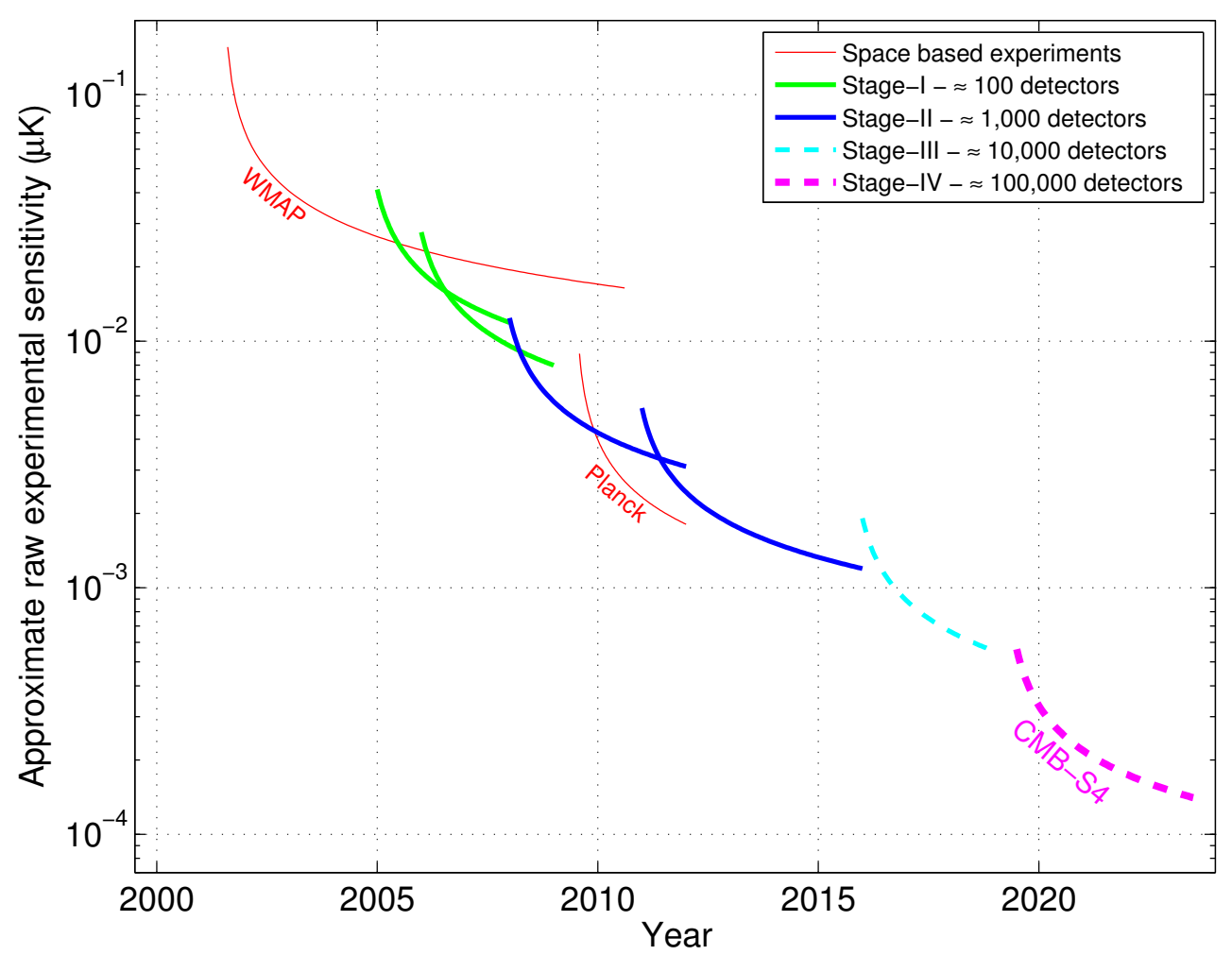

Figure 6. Plot illustrating the evolution of the raw sensitivity of CMB experiments, which scales as the total number of bolometers. Ground-based CMB experiments are classified into Stages with Stage II experiments having $O(1000)$ detectors, Stage III experiments having O(10,000) detectors, and a Stage IV experiment (such as CMB-S4) having $O(100,000)$ detectors.

consequence, the fundamental production unit for TES devices are arrays of detectors (see Fig. 8), an important attribute when considering the production of the 500,000 detectors required by CMB-S4. Second TES devices are low-impedance $(\leq 1 \Omega)$ and can be multiplexed with modern-day Superconducting QUantum Interference Device (SQUID) multiplexers [96, 97, 98]. Multiplexed readouts are important for operating large detector arrays at sub-Kelvin temperatures and are essential for CMB-S4. Lastly, TES detectors have been successfully deployed as focal planes at the forefront of CMB measurements.

The TES was invented by HEP for detecting Dark Matter and neutrinos. Its subsequent integration into CMB focal planes has enabled kilo-pixel arrays realizing the Stage II CMB program and ushering in an era of unprecedented sensitivity. TES-based CMB detectors are the favored technology among Stage II and proposed Stage III experiments, and have a clear path to the sensitivities required by CMB-S4. The ubiquity of TES detectors for CMB illustrates the direct connection between HEP-invented technology and CMB science.

\section{The CMB-S4 Experimental Program}

Delivering a half-million background-limited bolometers necessitates a change in the execution of the US ground-based CMB program. The current US program consists of a number of independent (primarily 

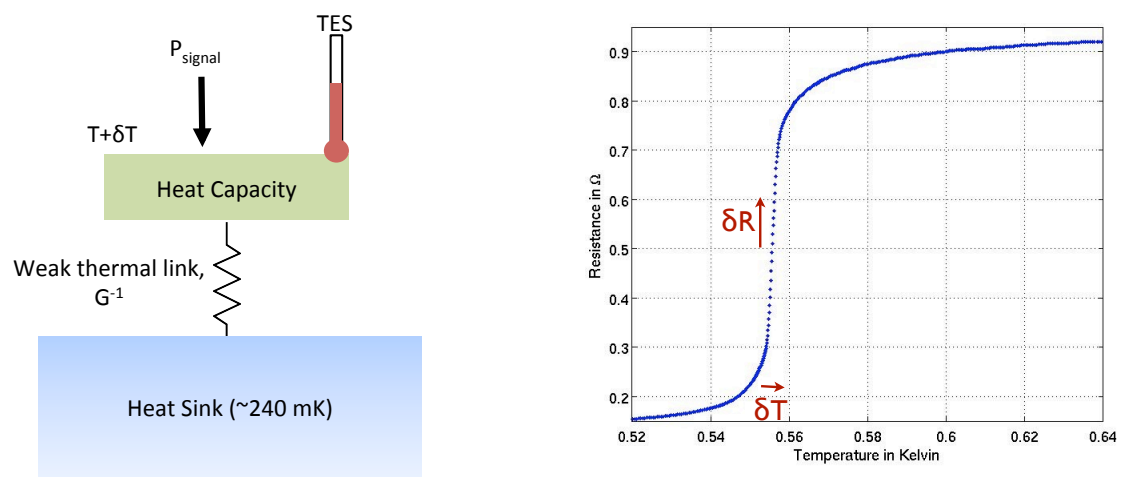

Figure 7. Left: Illustration of a thermal circuit for a typical Transition Edge Sensor (TES) detector highlighting the principles of signal detection. A weakly thermally sunk heat capacity absorbs power, $P_{\text {signal }}$, which is to be measured. Variations in the absorbed power change the heat capacity's temperature, which is measured by a TES operating under strong electro-thermal feedback. Right: Plot of resistance versus temperature for a typical TES illustrating the principles of negative electro-thermal feedback [95]. The TES is voltage biased into the middle of its superconducting-to-normal transition. Small changes in the TES temperature produce large changes in the TES resistance. Since the TES is voltage biased, an increase (or decrease) in the temperature produces an increase (or decrease) in the resistance leading to a decrease (or increase) in the Joule heating power supplied by the bias. This canceling effect corresponds to a strong negative electro-thermal feedback making the current through the TES nearly proportional to $P_{\text {signal }}$.

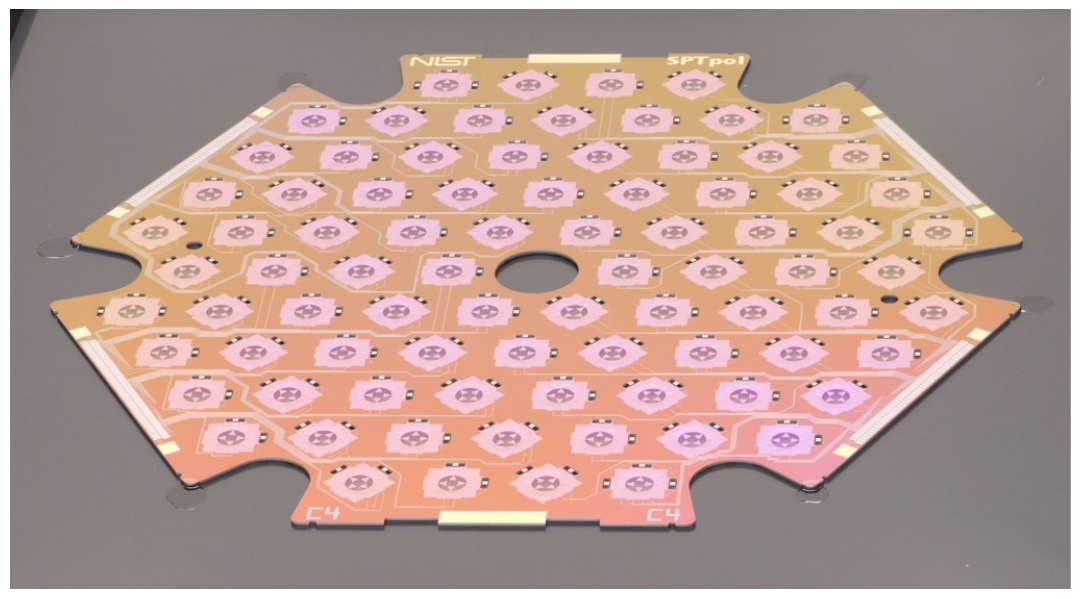

Figure 8. An example of a microfabricated array of TES bolometers, an 88 pixel, dual polarization TES bolometer array made at NIST for the SPTpol experiment. TES detectors are micro-machined from thin films deposited on silicon wafer substrates which means TES-based devices are fundamentally fabricated as arrays.

university) efforts, each focused on the development and delivery of their own instrument. The involvement of HEP in the current process has been through small investments targeted at specific technical contributions. Realizing CMB-S4 will require a radical change in this approach where HEP resources take the leading role. Of particular importance is an increased participation and support of national labs to provide resources 
which are unavailable to university groups. In particular, 1) leveraging micro-fabrication tools and expertise available at multi-purpose national labs is essential for the successful fabrication of the CMB-S4 detectors, and 2) supporting the computing infrastructure to support the greatly increased data rate, dataset size and analysis complexity.

The CMB-S4 experimental program will build on the success of Stage II and Stage III CMB experiments. It will be a coherent effort incorporating resources from both national labs and university groups.

The major instrumentation challenges are:

- Improved Production Reliability The favored technology for CMB-S4 are TES bolometers coupled through superconducting microstrip. Critical for the TES technology is reliable and optimal superconducting microstrip performance at millimeter wavelengths. Recent work on microstrip-coupled CMB detectors have demonstrated that it is possible to make superconducting microstrip which is virtually loss-less at the required frequencies [99]; however, the fabrication yield needs to be improved for CMBS4. Thus, one of the principle components of the CMB-S4 program is developing a reliable mass production process. Such work requires well maintained tooling, dedicated materials deposition, and understanding and control of all the materials dependent loss mechanisms.

- Increased Production Volume and Throughput Achieving $O(500,000)$ TES detectors demands new investment into TES array production resources. The required production throughput needs access to micro-fabrication resources with exclusive control of the thin film deposition systems. This exclusive access to microfabrication tooling falls squarely within the domain of national labs. Additionally, an extensive program of detector testing, characterization, and quality control is crucial for the mass production of 500,000 TES bolometers. This requirement will be met by establishing test facilities and organizing a quality assurance program among the universities and national labs.

- Multiplexed TES Readout Multiplexed TES readouts are required for implementing focal planes with more than 1000 detector elements and will continue to be an active component of the CMBS4 R\&D program. Modest improvements over existing fielded Stage-II multiplexer technology will be sufficient for the needs of CMB-S4. However, recent developments with microwave-based readout techniques for TES detetors may lead to new multiplexer technologies with broader applicability and lower cost, and could be synergistic with mKID readout development efforts.

- Large Cryogenic Optics The large size of the CMB-S4 focal planes together with the required sub-Kelvin operating temperature necessitates the development of new broad-band large aperture refractive optics which permit high throughput at millimeter wavelengths while blocking infrared thermal emission. Upcoming Stage-III experiments will serves as a proving ground for some new cryogenic optics technologies. CMB-S4 will build on these Stage-III accomplishments with the goal of developing manufacturing techniques to yield a large number of customized cryogenic optics with optimal performance.

The CMB-S4 program also presents equally challenging requirements in computing infrastructure and analysis tools. The analysis of a CMB dataset typically proceeds in sequence of steps:

- Map-making reduces the detectors' samples to maps of the temperature and polarization (typically split into $Q$ and $U$ modes) of the observed sky,

- Component separation extracts the CMB from any foreground contaminants in these maps, 
- Power spectrum estimation reduces the CMB maps to the six auto (TT, EE, BB) and cross $(T E, T B, E B)$ angular power spectra of the temperature and polarization (now expressed as $E$ and $B$ modes),

- Parameter estimation determines the likelihoods of the parameters of any chosen cosmological model given these power spectra.

This is essentially a series of data compressions, from the time- to the pixel- to the multipole- to the parameter-domain, and the methods we are able to employ to perform each of these steps will depend on their computational costs.

The CMB-S4 experiment presented here will field $O(500,000)$ detectors each sampling at $100 \mathrm{~Hz}$ for 5 years, during which it will repeatedly scan half of the sky at 3 arcminute resolution or better. Assuming a $70 \%$ duty cycle, this will yield a dataset with $\mathcal{N}_{t} \sim 6 \times 10^{15}$ time samples over $\mathcal{N}_{p} \sim 6 \times 10^{8}$ sky pixels. Note that the science goals of this experiment require 10,000 times as many observations per pixel as the current Planck satellite mission.

Under minimal assumptions, we can write simple Gaussian likelihoods for the maps given the data and the noise correlations in the time domain, and for the power spectra given the maps and the noise correlations in the pixel domain. However, the number of operations required first to construct the dense pixel domain noise correlation matrix, and then to use it to maximize the spectral likelihood, both scale as the cube of the number of pixels in the map; since $\mathcal{N}_{p}^{3} \sim 2 \times 10^{26}$ here, this approach is clearly unrealistic.

The alternative is the pseudo-spectral approach, where the power spectra are estimated from the maps ignoring their inhomogeneous noise, incomplete sky coverage, and any filtering applied to remove parasitic signals such as atmospheric emissions. The resulting pseudo-spectra are then corrected for the biases this introduces by applying a transfer function. Ideally this function is determined by Monte Carlo methods, simulating many realizations of the data and analyzing each in the same way as the real data. Comparing the simulations' known input spectra and derived output pseudo-spectra we can estimate the transfer function, which can then be inverted and applied to the real pseudo-spectra. Dominated by the iterative map-making - whether destriping or maximum likelihood - this analysis scales linearly with the numbers of realizations, iterations and samples, for an overall operation count of $\mathcal{N}_{r} \mathcal{N}_{i} \mathcal{N}_{t} \sim 6 \times 10^{21}$, assuming 10,000 realizations (for $1 \%$ uncertainties) each requiring 100 iterations.

While this is a daunting number, it is consistent with the exponential growth in the size of CMB datasets over the last 20 years (see Snowmass Cosmological Computing report). Mirroring as it does Moore's Law for computational capability, this growth constrains us to stay on the leading edge of high performance computing if we wish to realize the full scientific potential of the datasets we are gathering. Staying on that leading edge - wherever it may take us - represents the core challenge of CMB data analysis. To date this has necessitated following the evolution from simple increases in processor clock speed, to higher and higher core counts, to increasingly heterogenous accelerator-based systems. Beyond that, on the timescale of a CMB-S4 mission, we can only speculate, although we can be confident that exploiting the full capability of these systems will become more and more challenging as they become increasingly inhomogeneous, powerconstrained, and data-bound, and will require continued investment by HEP in the computational science underpinning these analyses. 


\section{The role of the national laboratories in CMB experiment}

CMB-S4 is the leading future CMB experiment. It is an ambitious undertaking where the primary technical challenge is one of scale, both in detectors and the resulting data set. For reference, current Stage-II instruments have focal planes with $O(1000)$ elements whereas CMB-S4 will have $O(500,000)$. To address the technical challenges of CMB-S4, HEP must re-envision its role in CMB experiments. Critical for the success of CMB-S4 is significant participation by national labs. This increased participation will be complemented with an organized program with university groups. The focus of the CMB-S4 technical R\&D will be the development of essential technology for the mass production and operation of the 500,000 TES detectors, and analysis of the resulting large data set.

CMB-S4 will have a profound impact on our understanding of High Energy Physics. It will provide the best path forward for investigating inflation, i.e., through the measurement of $B$-mode polarization of the CMB imprinted by inflationary gravitational waves, while also using CMB lensing to lead to measurement of the sum of the neutrino masses with an uncertainties of $16 \mathrm{meV}$. CMB-S4 will be the CMB experiment of the upcoming decade and it is critical for understanding cosmology and high energy physics in the post-Planck era. 


\section{References}

[1] Planck Collaboration Collaboration, P. Ade et al., "Planck 2013 results. XVI. Cosmological parameters," arXiv:1303.5076 [astro-ph.CO].

[2] S. Weinberg, "Goldstone Bosons as Fractional Cosmic Neutrinos," Phys.Rev.Lett. 110 (2013) 241301, arXiv:1305.1971 [astro-ph.CO].

[3] Z. Hou, C. Reichardt, K. Story, B. Follin, R. Keisler, et al., "Constraints on Cosmology from the Cosmic Microwave Background Power Spectrum of the 2500-square degree SPT-SZ Survey," arXiv:1212.6267 [astro-ph.CO].

[4] J. Lesgourgues and S. Pastor, "Massive neutrinos and cosmology," Phys.Rept. 429 (2006) 307-379, arXiv:astro-ph/0603494 [astro-ph].

[5] S. Das, B. D. Sherwin, P. Aguirre, J. W. Appel, J. R. Bond, et al., "Detection of the Power Spectrum of Cosmic Microwave Background Lensing by the Atacama Cosmology Telescope," Phys.Rev.Lett. 107 (2011) 021301, arXiv:1103.2124 [astro-ph.CO].

[6] A. van Engelen, R. Keisler, O. Zahn, K. A. Aird, B. A. Benson, L. E. Bleem, J. E. Carlstrom, C. L. Chang, H. M. Cho, T. M. Crawford, A. T. Crites, T. de Haan, M. A. Dobbs, J. Dudley, E. M. George, N. W. Halverson, G. P. Holder, W. L. Holzapfel, S. Hoover, Z. Hou, J. D. Hrubes, M. Joy, L. Knox, A. T. Lee, E. M. Leitch, M. Lueker, D. Luong-Van, J. J. McMahon, J. Mehl, S. S. Meyer, M. Millea, J. J. Mohr, T. E. Montroy, T. Natoli, S. Padin, T. Plagge, C. Pryke, C. L. Reichardt, J. E. Ruhl, J. T. Sayre, K. K. Schaffer, L. Shaw, E. Shirokoff, H. G. Spieler, Z. Staniszewski, A. A. Stark, K. Story, K. Vanderlinde, J. D. Vieira, and R. Williamson, "A Measurement of Gravitational Lensing of the Microwave Background Using South Pole Telescope Data," Astrophys. J. 756 (Sept., 2012) 142, arXiv: 1202.0546 [astro-ph.CO].

[7] S. Das, T. Louis, M. R. Nolta, G. E. Addison, E. S. Battistelli, et al., "The Atacama Cosmology Telescope: Temperature and Gravitational Lensing Power Spectrum Measurements from Three Seasons of Data," arXiv:1301.1037 [astro-ph.CO].

[8] G. Holder, M. Viero, O. Zahn, K. Aird, B. Benson, et al., "A Cosmic Microwave Background Lensing Mass Map and Its Correlation with the Cosmic Infrared Background," Astrophys.J. 771 (2013) L16, arXiv:1303.5048 [astro-ph.CO].

[9] Planck Collaboration Collaboration, P. Ade et al., "Planck 2013 results. XVII. Gravitational lensing by large-scale structure," arXiv:1303.5077 [astro-ph.CO].

[10] J. Geach, R. Hickox, L. Bleem, M. Brodwin, G. Holder, et al., "A direct measurement of the linear bias of mid-infrared-selected quasars at $\mathrm{z}^{\sim} 1$ using cosmic microwave background lensing," arXiv:1307.1706 [astro-ph.CO].

[11] D. Hanson, S. Hoover, A. Crites, P. Ade, K. Aird, et al., "Detection of B-mode Polarization in the Cosmic Microwave Background with Data from the South Pole Telescope," arXiv:1307.5830 [astro-ph.CO].

[12] C. Blake, S. Brough, M. Colless, C. Contreras, W. Couch, S. Croom, D. Croton, T. M. Davis, M. J. Drinkwater, K. Forster, D. Gilbank, M. Gladders, K. Glazebrook, B. Jelliffe, R. J. Jurek, I.-h. Li, B. Madore, D. C. Martin, K. Pimbblet, G. B. Poole, M. Pracy, R. Sharp, E. Wisnioski, D. Woods, T. K. Wyder, and H. K. C. Yee, "The WiggleZ Dark Energy Survey: joint measurements of the expansion and growth history at z i 1," Mon.Not.Roy.Astron.Soc. 425 (Sept., 2012) 405-414, arXiv:1204. 3674 [astro-ph.CO]. 
[13] L. Anderson, E. Aubourg, S. Bailey, D. Bizyaev, M. Blanton, et al., "The clustering of galaxies in the SDSS-III Baryon Oscillation Spectroscopic Survey: Baryon Acoustic Oscillations in the Data Release 9 Spectroscopic Galaxy Sample," Mon.Not.Roy.Astron.Soc. 427 no. 4, (2013) 3435-3467, arXiv:1203.6594 [astro-ph.CO].

[14] N. G. Busca, T. Delubac, J. Rich, S. Bailey, A. Font-Ribera, et al., "Baryon Acoustic Oscillations in the Ly- $\alpha$ forest of BOSS quasars," Astron.Astrophys. 552 (2013) A96, arXiv:1211.2616 [astro-ph.C0].

[15] A. Slosar, V. Irsic, D. Kirkby, S. Bailey, N. G. Busca, et al., "Measurement of Baryon Acoustic Oscillations in the Lyman-alpha Forest Fluctuations in BOSS Data Release 9," JCAP 1304 (2013) 026, arXiv:1301.3459 [astro-ph.CO].

[16] LSND Collaboration Collaboration, A. Aguilar-Arevalo et al., "Evidence for neutrino oscillations from the observation of anti-neutrino(electron) appearance in a anti-neutrino(muon) beam," Phys.Rev. D64 (2001) 112007, arXiv:hep-ex/0104049 [hep-ex].

[17] MiniBooNE Collaboration Collaboration, A. Aguilar-Arevalo et al., "Event Excess in the MiniBooNE Search for $\bar{\nu}_{\mu} \rightarrow \bar{\nu}_{e}$ Oscillations," Phys.Rev.Lett. 105 (2010) 181801, arXiv:1007.1150 [hep-ex].

[18] J. Kopp, M. Maltoni, and T. Schwetz, "Are there sterile neutrinos at the eV scale?," Phys.Rev.Lett. 107 (2011) 091801, arXiv:1103.4570 [hep-ph].

[19] C. Giunti and M. Laveder, "3+1 and 3+2 Sterile Neutrino Fits," Phys.Rev. D84 (2011) 073008, arXiv:1107.1452 [hep-ph].

[20] G. Mention, M. Fechner, T. Lasserre, T. Mueller, D. Lhuillier, et al., "The Reactor Antineutrino Anomaly," Phys.Rev. D83 (2011) 073006, arXiv:1101.2755 [hep-ex].

[21] P. Huber, "On the determination of anti-neutrino spectra from nuclear reactors," Phys.Rev. C84 (2011) 024617, arXiv:1106.0687 [hep-ph]. Erratum-ibid. C85 (2012) 029901.

[22] G. Fogli, E. Lisi, A. Marrone, D. Montanino, A. Palazzo, et al., "Global analysis of neutrino masses, mixings and phases: entering the era of leptonic CP violation searches," Phys.Rev. D86 (2012) 013012, arXiv: 1205.5254 [hep-ph].

[23] G. Mangano, G. Miele, S. Pastor, T. Pinto, O. Pisanti, et al., "Relic neutrino decoupling including flavor oscillations," Nucl.Phys. B729 (2005) 221-234, arXiv:hep-ph/0506164 [hep-ph].

[24] J. Bernstein, Kinetic theory in the expanding universe. Cambridge and New York, Cambridge University Press, 1988.

[25] Z. Hou, R. Keisler, L. Knox, M. Millea, and C. Reichardt, "How massless neutrinos affect the cosmic microwave background damping tail," Phys. Rev. D87 no. 8, (Apr., 2013) 083008, arXiv:1104. 2333 [astro-ph.CO].

[26] WMAP Collaboration, C. Bennett et al., "Nine-Year Wilkinson Microwave Anisotropy Probe (WMAP) Observations: Final Maps and Results," arXiv:1212.5225 [astro-ph.CO].

[27] K. Story, C. Reichardt, Z. Hou, R. Keisler, K. Aird, et al., "A Measurement of the Cosmic Microwave Background Damping Tail from the 2500-square-degree SPT-SZ survey," arXiv:1210.7231 [astro-ph.CO].

[28] G. Jungman, M. Kamionkowski, A. Kosowsky, and D. N. Spergel, "Cosmological parameter determination with microwave background maps," Phys.Rev. D54 (1996) 1332-1344, arXiv:astro-ph/9512139 [astro-ph]. 
[29] D. J. Eisenstein and W. Hu, "Power spectra for cold dark matter and its variants," Astrophys.J. 511 (1997) 5, arXiv:astro-ph/9710252 [astro-ph].

[30] W. Hu, D. J. Eisenstein, and M. Tegmark, "Weighing neutrinos with galaxy surveys," Phys.Rev.Lett. 80 (1998) 5255-5258, arXiv:astro-ph/9712057 [astro-ph].

[31] S. Dodelson, E. Gates, and A. Stebbins, "Cold + hot dark matter and the cosmic microwave background," Astrophys.J. 467 (1996) 10-18, arXiv:astro-ph/9509147 [astro-ph].

[32] W. Hu and S. Dodelson, "Cosmic microwave background anisotropies," Ann.Rev.Astron.Astrophys. 40 (2002) 171-216, arXiv:astro-ph/0110414 [astro-ph].

[33] K. S. Dawson et al., "The Baryon Oscillation Spectroscopic Survey of SDSS-III," Astronomical Journal 145 (2013) 10, arXiv:1208.0022 [astro-ph.CO].

[34] G.-B. Zhao, S. Saito, W. J. Percival, A. J. Ross, F. Montesano, et al., "The clustering of galaxies in the SDSS-III Baryon Oscillation Spectroscopic Survey: weighing the neutrino mass using the galaxy power spectrum of the CMASS sample," arXiv:1211.3741 [astro-ph.CO].

[35] S. Riemer-Srensen, D. Parkinson, and T. M. Davis, "Combining Planck with Large Scale Structure gives strong neutrino mass constraint," arXiv:1306.4153 [astro-ph.CO].

[36] M. Zaldarriaga and U. Seljak, "Gravitational lensing effect on cosmic microwave background polarization," Phys.Rev. D58 (1998) 023003, arXiv:astro-ph/9803150 [astro-ph].

[37] V. K. Narayanan, A. A. Berlind, and D. H. Weinberg, "Locally biased galaxy formation and large scale structure," Astrophys.J. 528 (2000) 1-20, arXiv:astro-ph/9812002 [astro-ph].

[38] SDSS Collaboration Collaboration, M. Tegmark et al., "The 3-D power spectrum of galaxies from the SDSS," Astrophys.J. 606 (2004) 702-740, arXiv:astro-ph/0310725 [astro-ph].

[39] P. McDonald and U. Seljak, "How to measure redshift-space distortions without sample variance," JCAP 0910 (2009) 007, arXiv:0810.0323 [astro-ph].

[40] A. Font-Ribera, P. McDonald, N. Mostek, B. A. Reid, H.-J. Seo, et al., "DESI and other dark energy experiments in the era of neutrino mass measurements," arXiv:1308.4164 [astro-ph.CO].

[41] N. Kaiser, "Weak gravitational lensing of distant galaxies," Astrophys.J. 388 (1992) 272.

[42] B. Jain and U. Seljak, "Cosmological model predictions for weak lensing: Linear and nonlinear regimes," Astrophys.J. 484 (1997) 560, arXiv:astro-ph/9611077 [astro-ph].

[43] W. Hu, "Power spectrum tomography with weak lensing," Astrophys.J. 522 (1999) L21-L24, arXiv:astro-ph/9904153 [astro-ph].

[44] A. R. Cooray, "Weighing neutrinos: Weak lensing approach," Astron.Astrophys. 348 (1999) 31, arXiv:astro-ph/9904246 [astro-ph].

[45] K. N. Abazajian and S. Dodelson, "Neutrino mass and dark energy from weak lensing," Phys.Rev.Lett. 91 (2003) 041301, arXiv:astro-ph/0212216 [astro-ph].

[46] L. Wolz, M. Kilbinger, J. Weller, and T. Giannantonio, "On the Validity of Cosmological Fisher Matrix Forecasts," JCAP 1209 (2012) 009, arXiv:1205.3984 [astro-ph.CO].

[47] S. Khedekar and S. Majumdar, "Cosmology with the largest galaxy cluster surveys: Going beyond Fisher matrix forecasts," JCAP 1302 (2013) 030, arXiv:1210.5586 [astro-ph.Co]. 
[48] EUCLID Collaboration Collaboration, R. Laureijs et al., "Euclid Definition Study Report," arXiv:1110.3193 [astro-ph.CO].

[49] K. M. Smith, A. Cooray, S. Das, O. Doré, D. Hanson, C. Hirata, M. Kaplinghat, B. Keating, M. Loverde, N. Miller, G. Rocha, M. Shimon, and O. Zahn, "Gravitational Lensing," in American Institute of Physics Conference Series, S. Dodelson, D. Baumann, A. Cooray, J. Dunkley, A. Fraisse, M. G. Jackson, A. Kogut, L. Krauss, M. Zaldarriaga, and K. Smith, eds., vol. 1141 of American Institute of Physics Conference Series, pp. 121-178. June, 2009. arXiv:0811.3916.

[50] LBNE Collaboration Collaboration, C. Adams et al., "Scientific Opportunities with the LongBaseline Neutrino Experiment," arXiv:1307.7335 [hep-ex].

[51] S. Joudaki and M. Kaplinghat, "Dark Energy and Neutrino Masses from Future Measurements of the Expansion History and Growth of Structure," Phys.Rev. D86 (2012) 023526, arXiv:1106.0299 [astro-ph.CO].

[52] M. Kaplinghat, L. Knox, and Y.-S. Song, "Determining neutrino mass from the CMB alone," Phys.Rev.Lett. 91 (2003) 241301, arXiv:astro-ph/0303344 [astro-ph].

[53] Planck Collaboration, P. Ade et al., "Planck 2013 Results. XXIV. Constraints on primordial nonGaussianity," arXiv:1303.5084 [astro-ph.CO].

[54] LSST Collaboration Collaboration, Z. Ivezic, J. Tyson, R. Allsman, J. Andrew, and R. Angel, "LSST: from Science Drivers to Reference Design and Anticipated Data Products," arXiv:0805.2366 [astro-ph].

[55] D. Spergel, N. Gehrels, J. Breckinridge, M. Donahue, A. Dressler, et al., "Wide-Field InfraRed Survey Telescope-Astrophysics Focused Telescope Assets WFIRST-AFTA Final Report," arXiv:1305.5422 [astro-ph.IM].

[56] C. Carbone, L. Verde, Y. Wang, and A. Cimatti, "Neutrino constraints from future nearly all-sky spectroscopic galaxy surveys," JCAP 1103 (2011) 030, arXiv:1012.2868 [astro-ph.C0].

[57] J. Hamann, S. Hannestad, and Y. Y. Wong, "Measuring neutrino masses with a future galaxy survey," JCAP 1211 (2012) 052, arXiv:1209.1043 [astro-ph.CO].

[58] C. Carbone, C. Fedeli, L. Moscardini, and A. Cimatti, "Measuring the neutrino mass from future wide galaxy cluster catalogues," JCAP 1203 (2012) 023, arXiv:1112.4810 [astro-ph.CO].

[59] T. Basse, O. E. Bjaelde, J. Hamann, S. Hannestad, and Y. Y. Y. Wong, "Dark energy and neutrino constraints from a future EUCLID-like survey," arXiv:1304.2321 [astro-ph.CO].

[60] U. Seljak, A. Slosar, and P. McDonald, "Cosmological parameters from combining the Lymanalpha forest with CMB, galaxy clustering and SN constraints," JCAP 0610 (2006) 014, arXiv:astro-ph/0604335 [astro-ph].

[61] M. Viel, M. G. Haehnelt, and V. Springel, "The effect of neutrinos on the matter distribution as probed by the Intergalactic Medium," JCAP 1006 (2010) 015, arXiv:1003.2422 [astro-ph.CO].

[62] S. Gratton, A. Lewis, and G. Efstathiou, "Prospects for Constraining Neutrino Mass Using Planck and Lyman-Alpha Forest Data," Phys.Rev. D77 (2008) 083507, arXiv:0705.3100 [astro-ph].

[63] E. Switzer, K. Masui, K. Bandura, L. M. Calin, T. C. Chang, et al., "Determination of z 0.8 neutral hydrogen fluctuations using the $21 \mathrm{~cm}$ intensity mapping auto-correlation," arXiv:1304.3712 [astro-ph.CO]. 
[64] M. F. Morales and J. S. B. Wyithe, "Reionization and Cosmology with $21 \mathrm{~cm}$ Fluctuations," Ann.Rev.Astron.Astrophys. 48 (2010) 127-171, arXiv:0910.3010 [astro-ph.CO].

[65] Y. Mao, M. Tegmark, M. McQuinn, M. Zaldarriaga, and O. Zahn, "How accurately can $21 \mathrm{~cm}$ tomography constrain cosmology?," Phys.Rev. D78 (2008) 023529, arXiv:0802.1710 [astro-ph].

[66] S. Wang, Z. Haiman, W. Hu, J. Khoury, and M. May, "Weighing neutrinos with galaxy cluster surveys," Phys.Rev.Lett. 95 (2005) 011302, arXiv:astro-ph/0505390 [astro-ph].

[67] K. Abazajian, E. Calabrese, A. Cooray, F. De Bernardis, S. Dodelson, et al., "Cosmological and Astrophysical Neutrino Mass Measurements," Astropart.Phys. 35 (2011) 177-184, arXiv:1103.5083 [astro-ph.CO].

[68] W. H. Kinney, "How to fool cosmic microwave background parameter estimation," Phys.Rev. D63 (2001) 043001, arXiv:astro-ph/0005410 [astro-ph].

[69] D. K. Hazra, A. Shafieloo, and T. Souradeep, "Cosmological parameter estimation with free-form primordial power spectrum," arXiv:1303.5336 [astro-ph.CO].

[70] B. A. Bassett, S. Tsujikawa, and D. Wands, "Inflation dynamics and reheating," Rev.Mod.Phys. 78 (2006) 537-589, arXiv:astro-ph/0507632 [astro-ph].

[71] M. Ishak, A. Upadhye, and D. N. Spergel, "Probing cosmic acceleration beyond the equation of state: Distinguishing between dark energy and modified gravity models," Phys.Rev. D74 (2006) 043513, arXiv: astro-ph/0507184 [astro-ph].

[72] K. Heitmann, E. Lawrence, J. Kwan, S. Habib, and D. Higdon, "The Coyote Universe Extended: Precision Emulation of the Matter Power Spectrum," arXiv:1304.7849 [astro-ph.CO].

[73] H. Zhan and L. Knox, "Effect of hot baryons on the weak-lensing shear power spectrum," Astrophys.J. 616 (2004) L75-L78, arXiv:astro-ph/0409198 [astro-ph].

[74] Y. Jing, P. Zhang, W. Lin, L. Gao, and V. Springel, "The influence of baryons on the clustering of matter and weak lensing surveys," Astrophys.J. 640 (2006) L119-L122, arXiv:astro-ph/0512426 [astro-ph].

[75] A. R. Zentner, D. H. Rudd, and W. Hu, "Self Calibration of Tomographic Weak Lensing for the Physics of Baryons to Constrain Dark Energy," Phys.Rev. D77 (2008) 043507, arXiv:0709.4029 [astro-ph].

[76] K. Abazajian, E. R. Switzer, S. Dodelson, K. Heitmann, and S. Habib, "The Nonlinear cosmological matter power spectrum with massive neutrinos. 1. The Halo model," Phys.Rev. D71 (2005) 043507, arXiv: astro-ph/0411552 [astro-ph].

[77] J. Brandbyge, S. Hannestad, T. Haugbolle, and B. Thomsen, "The Effect of Thermal Neutrino Motion on the Non-linear Cosmological Matter Power Spectrum," JCAP 0808 (2008) 020, arXiv:0802.3700 [astro-ph].

[78] F. Villaescusa-Navarro, S. Bird, C. Pena-Garay, and M. Viel, "Non-linear evolution of the cosmic neutrino background," JCAP 1303 (2013) 019, arXiv:1212.4855 [astro-ph.C0].

[79] M. LoVerde and M. Zaldarriaga, "Neutrino clustering around spherical dark matter halos," arXiv:1310.6459 [astro-ph.CO].

[80] G. Rossi, N. Palanque-Delabrouille, A. Borde, M. Viel, C. Yeche, et al., "A Suite of Hydrodynamical Simulations for the Lyman-Alpha Forest with Massive Neutrinos," arXiv:1401.6464 [astro-ph.C0]. 
[81] W. Hu, M. M. Hedman, and M. Zaldarriaga, "Benchmark parameters for CMB polarization experiments," Phys.Rev. D67 (2003) 043004, arXiv:astro-ph/0210096 [astro-ph].

[82] C. MacTavish, P. Ade, E. Battistelli, S. Benton, R. Bihary, et al., "Spider Optimization: Probing the Systematics of a Large Scale B-Mode Experiment," arXiv:0710.0375 [astro-ph].

[83] Y. Takahashi, P. Ade, D. Barkats, J. Battle, E. Bierman, et al., "Characterization of the BICEP Telescope for High-Precision Cosmic Microwave Background Polarimetry," Astrophys.J. 711 (2010) 1141-1156, arXiv:0906.4069 [astro-ph.C0].

[84] J. Vieira, T. Crawford, E. Switzer, P. Ade, K. Aird, et al., "Extragalactic millimeter-wave sources in South Pole Telescope survey data: source counts, catalog, and statistics for an 87 square-degree field," Astrophys.J. 719 (2010) 763-783, arXiv:0912.2338 [astro-ph.CO].

[85] T. Marriage, J. Juin, Y.-T. Lin, D. Marsden, M. Nolta, et al., "The Atacama Cosmology Telescope: Extragalactic Sources at $148 \mathrm{GHz}$ in the 2008 Survey," Astrophys.J. 731 (2011) 100, arXiv:1007.5256 [astro-ph.CO].

[86] Planck Collaboration Collaboration, P. Ade et al., "Planck Early Results. VII. The Early Release Compact Source Catalog," arXiv:1101.2041 [astro-ph.CO].

[87] R. Battye, I. Browne, M. Peel, N. Jackson, and C. Dickinson, "Statistical properties of polarized radio sources at high frequency and their impact on CMB polarization measurements," Mon.Not.Roy.Astron.Soc. 413 (2010) 132-148, arXiv:1003.5846 [astro-ph.C0].

[88] M. Seiffert, C. Borys, D. Scott, and M. Halpern, "An upper limit to polarized submillimetre emission in Arp 220," Mon.Not.Roy.Astron.Soc. 374 (2007) 409-414, arXiv:astro-ph/0610485 [astro-ph].

[89] 2dFGRS Collaboration Collaboration, S. Cole et al., "The 2dF Galaxy Redshift Survey: Powerspectrum analysis of the final dataset and cosmological implications," Mon.Not.Roy.Astron.Soc. 362 (2005) 505-534, arXiv:astro-ph/0501174 [astro-ph].

[90] D. J. Eisenstein, H.-j. Seo, and . White, Martin J., "On the Robustness of the Acoustic Scale in the Low-Redshift Clustering of Matter," Astrophys.J. 664 (2007) 660-674, arXiv:astro-ph/0604361 [astro-ph].

[91] A. J. Ross, S. Ho, A. J. Cuesta, R. Tojeiro, W. J. Percival, et al., "Ameliorating Systematic Uncertainties in the Angular Clustering of Galaxies: A Study using SDSS-III," Mon.Not.Roy.Astron.Soc. 417 (2011) 1350, arXiv:1105.2320 [astro-ph.CO].

[92] BOSS Collaboration Collaboration, A. J. Ross et al., "The clustering of galaxies in the SDSS-III Baryon Oscillation Spectroscopic Survey: Analysis of potential systematics," Mon.Not.Roy.Astron.Soc. 424 (2012) 564, arXiv:1203.6499 [astro-ph.CO].

[93] S. Ho, A. Cuesta, H.-J. Seo, R. de Putter, A. J. Ross, et al., "Clustering of Sloan Digital Sky Survey III Photometric Luminous Galaxies: The Measurement, Systematics and Cosmological Implications," Astrophys.J. 761 (2012) 14, arXiv:1201.2137 [astro-ph.CO].

[94] A. R. Pullen and C. M. Hirata, "Systematic effects in large-scale angular power spectra of photometric quasars and implications for constraining primordial nongaussianity," arXiv:1212.4500 [astro-ph.CO].

[95] K. D. Irwin, "An application of electrothermal feedback for high resolution cryogenic particle detection," Applied Physics Letters 66 no. 15, (1995) 1998-2000. 
[96] T. M. Lanting, K. Arnold, H.-M. Cho, J. Clarke, M. Dobbs, W. Holzapfel, A. T. Lee, M. Lueker, P. L. Richards, A. D. Smith, and H. G. Spieler, "Frequency-domain readout multiplexing of transition-edge sensor arrays," Nuclear Instruments and Methods in Physics Research A 559 (Apr., 2006) 793-795.

[97] K. D. Irwin, L. R. Vale, N. E. Bergren, S. Deiker, E. N. Grossman, G. C. Hilton, S. W. Nam, C. D. Reintsema, D. A. Rudman, and M. E. Huber, "Time-division SQUID multiplexers," Low Temperature Detectors 605 (Feb., 2002) 301-304.

[98] K. D. Irwin and K. W. Lehnert, "Microwave SQUID multiplexer," Applied Physics Letters 85 (Sept., 2004) 2107.

[99] J. W. Henning, P. Ade, K. A. Aird, J. E. Austermann, J. A. Beall, D. Becker, B. A. Benson, L. E. Bleem, J. Britton, J. E. Carlstrom, C. L. Chang, H.-M. Cho, T. M. Crawford, A. T. Crites, A. Datesman, T. de Haan, M. A. Dobbs, W. Everett, A. Ewall-Wice, E. M. George, N. W. Halverson, N. Harrington, G. C. Hilton, W. L. Holzapfel, J. Hubmayr, K. D. Irwin, M. Karfunkle, R. Keisler, J. Kennedy, A. T. Lee, E. Leitch, D. Li, M. Lueker, D. P. Marrone, J. J. McMahon, J. Mehl, S. S. Meyer, J. Montgomery, T. E. Montroy, J. Nagy, T. Natoli, J. P. Nibarger, M. D. Niemack, V. Novosad, S. Padin, C. Pryke, C. L. Reichardt, J. E. Ruhl, B. R. Saliwanchik, J. T. Sayre, K. K. Schaffer, E. Shirokoff, K. Story, C. Tucker, K. Vanderlinde, J. D. Vieira, G. Wang, R. Williamson, V. Yefremenko, K. W. Yoon, and E. Young, "Feedhorn-coupled tes polarimeter camera modules at $150 \mathrm{ghz}$ for $\mathrm{cmb}$ polarization measurements with sptpol,". +http://dx.doi.org/10.1117/12.927172. 